\title{
Il male in sé e il nulla in Proclo
}

DO।: $10.14746 / p e a .2020 .1 .6$

VALERIO NAPOLI / independent researcher/

Dedico questo mio modesto scritto, con affetto e gratitudine, alla memoria di mia madre Maria Salemi (1944-2019), che ha sempre alimentato e arricchito la mia vita con straordinario Amore materno

\section{Introduzione}

Nella sua teoria del male Proclo pone la distinzione tra il male in sé, concepito come totalmente privo di commistione con il bene, e il male mescolato al bene. Mentre il male in sé, che potremmo chiamare il male assoluto, è dichiarato non esistente, il male mescolato al bene, che potremmo chiamare il male relativo, è presentato invece come annoverabile 
tra le cose esistenti ${ }^{1}$. Sulla base di questa distinzione, Proclo sostiene la tesi dell'esistenza del male, nell'ambito di una visione filosofica incentrata sull'istanza monistica della derivazione e della dipendenza di tutte le cose da un unico principio primo, il semplicemente uno, il quale è assunto come identico al bene primo, con implicazioni che comportano la negazione di qualsivoglia forma di contrapposizione protologica tra il bene e il male e di ipostatizzazione del male. In questo quadro speculativo si delinea la nota concezio-

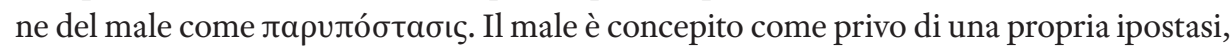
come esistente non in sé, bensì in altro e in virtù di altro, in forza del bene, nella modalità di un'esistenza collaterale e parassitaria, accidentale e avventizia, priva di cause proprie e principali, riscontrabile soltanto nelle anime particolari e nei corpi particolari. Così concepito, il male è posto come privazione parziale di bene, cioè come privazione di qualche determinato bene in una data realtà che resta pur sempre partecipe del bene e radicata nel bene. In tal modo, il male esiste nel suo essere mescolato al bene. Il male in sé, invece, in quanto totalmente non commisto al bene, è dichiarato meramente non esistente. A questo riguardo, è significativo il fatto che nel De malorum subsistentia la distinzione tra il male in sé e il male commisto al bene sia formulata, sul piano concettuale, in corrispondenza della distinzione di fondo tra il non-essente assoluto, il quale è assunto come nozione univoca che indica la totale assenza di esistenza, e il non-essente relativo, il quale si configura come esistente. Per un verso, infatti, il male assoluto è accostato al nonessente assoluto, cioè al nulla; per altro verso, il male commisto al bene è pensato, a suo modo, con riferimento al non-essente relativo, visto, oltre che nel suo generico statuto esistenziale, nella sua specifica articolazione tipologica consistente nella privazione.

In questo scenario Proclo afferma in varie occasioni che il male in sé è «al di là anche

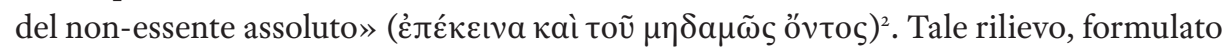
nell'ambito della prospettazione di una corrispondenza inversa tra il male in sé e il bene primo $^{3}$, appare pienamente comprensibile sulla base di una lettura della concezione procliana del male alla luce di taluni snodi concettuali della dottrina della multivocità del non-essente, la quale, nei suoi vari aspetti, riveste una particolare importanza nel pensiero del nostro filosofo. Nelle pagine che seguono intendo mostrare che in Proclo,

\footnotetext{
${ }^{1}$ Per l'uso delle espressioni di "male assoluto" e "male relativo" per indicare le due diverse concezioni del male in questo contesto, cfr. per es. Montoneri (1986: XV-XVI).

2 Cfr. Procl. in Ti. I 374, 14-15 Diehl; Procl. Theol.Plat. I 18, 86, 20-21 Saffrey, Westerink; Procl. DMS 3, 3-4 (espressione equivalente) e 9, 7-9 Isaac. In Proclo l'espressione tò $\mu \eta \delta \alpha \mu \tilde{\omega} \varsigma$ őv $v$, "ciò che non è in alcun modo", "l'assolutamente non-essente", di derivazione platonica (cfr. spec. Pl. Soph. 237b7-8 Burnet), indica propriamente

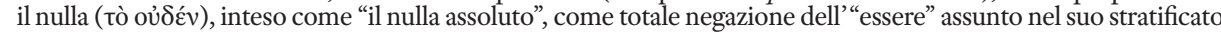
e onnicomprensivo senso esistenziale, dunque come cifra della totale assenza di qualsivolgia ordine e modalità di esistenza. A questo proposito, ritengo anche che nel lessico del nulla Proclo usi i due morfermi negativi oủ e $\mu$ í in modo sostanzialmente equivalente e intercambiabile, con un medesimo valore "oggettivo". In queste pagine traduco tò $\mu \eta \delta \alpha \mu \tilde{\omega}$ ऽ ôv con «il non-essente assoluto»; con l'espressione "il nulla" intendo riferirmi al non-essente assoluto.

${ }^{3}$ Il bene primo - espressione che in queste pagine utilizzo come adattamento di le prime bonum ( áyatóv, "il bene in senso primario") che ricorre nel testo (cfr. Procl. DMS 51, 9; Strobel 2014: 970, 51, 5-6) - è, come ho accennato, il principio primo di tutte le cose, il bene sovraessenziale, assunto nella sua trascendenza rispetto al bene formale e al bene in noi (cfr. Procl. in R. I 269, 4 ss.), e visto come la causa prima di tutte le cose e di ogni bene.
} 
la delineazione del male in sé come «al di là anche del non-essente assoluto», inquadrata nella sua peculiare trama argomentativa e decifrata nella sua pregnante valenza concettuale, lungi dal prospettare una distinzione e una divaricazione tra il male in sé e il nonessente assoluto, è mirata a stabilire in modo lampante e incontrovertibile la fattuale coincidenza di questi due termini e a ribadire la tesi dell'impossibilità dell'esistenza del male assoluto, a complemento della concomitante tesi della necessaria esistenza del male commisto al bene, un'esistenza che lo Scolarca considera come conciliabile con l'ordinamento agatologico della realtà e perfino funzionale alla configurazione di quest'ulti-

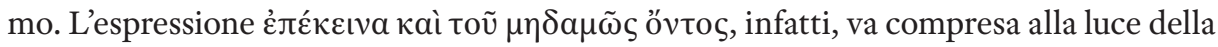
configurazione della nozione del non-essente assoluto - cifra della totale assenza difettiva di qualsivoglia ordine e tipo di esistenza -, che in Proclo si rivela "intrascendibile" e che, come vedremo, rispetto al principio primo di tutte le cose (l'uno-bene) e ai suoi effetti (l'unità-bontà), manifesta sul versante henologico uno statuto negativo-difettivo analogo a quello che il male assoluto mostra sul versante agatologico. Si profila, così, una radicale riduzione del male assoluto al nulla, la quale, per altro verso, permette di registrare in aree dell'articolato orizzonte dell'esistente il male mescolato al bene, in termini che Proclo considera come coerenti con le istanze del suo monismo agatologico.

\section{Il male in sé e il bene sovraessenziale. Linee di una corrispondenza inversa}

Nel De malorum subsistentia la delineazione del male in sé come «al di là anche del non-essente assoluto ${ }^{4}$ ricorre due volte, entrambe nella parte iniziale dello scritto, dedicata alla trattazione della prima questione presa in esame, se il male esista o no ${ }^{5}$. Proclo, dopo un'esposizione di taluni argomenti a favore di ciascuna delle due tesi, risolve la questione con il superamento della rigida contrapposizione delle due tesi - incentrate l'una sulla radicale negazione dell'esistenza del male e l'altra sull'affermazione dell'esistenza ipostatica del male - e con la formulazione della tesi che prospetta l'impossibilità dell'esistenza del male totalmente separato dal bene e la necessità dell'esistenza del male mescolato al bene. La prima occorrenza della presentazione del male come «al di là anche del non-essente assoluto» si registra nel $\$ 3$ dell'opusculum, in cui Proclo, tra gli argomenti a supporto della tesi della non esistenza del male, ne include uno che è basato sulla corrispondenza inversa del male al bene primo, al quale esso, sul piano concettuale, si contrappone. Va notato che in questo caso il male è assunto in termini ancora indifferenziati, a prescindere dalla distinzione tra il male puro e il male commisto al bene, la quale sarà stabilita successivamente come elemento dirimente per la soluzione della questione posta.

${ }^{4}$ Questa espressione, con la trama argomentativa in cui è inserita, è riconducibile a Siriano; cfr. Festugière (2006: 235); Opsomer, Steel (2003: $106 \mathrm{nt}$. 12). Sulla dipendenza della dottrina procliana del male dal pensiero di Siriano cfr. d'Hoine (2011: 77-78 e nota 12). Sulle fonti della dottrina procliana del male si vedano anche Bechtle (1999); Opsomer, Steel (1999); Cardullo (2017: 58-61).

5 Cfr. Procl. DMS 1, 15-16; 2, 1-3. 
Bisogna però rilevare che in questo argomento, il male, secondo una specificazione implicita nella trama concettuale in cui è presentato, è posto di fatto in termini di assolutezza, come male in sé, proprio perché esso è accostato non a un qualche bene, bensì al bene primo, che è, potremmo dire, il bene assoluto. Questa corrispondenza permette di porre il male in relazione al non-essente assoluto:

Et quid oportet dicere multa? Si enim ultra le esse le unum et quam dicimus boni naturam, ultra ipsum non esse malum - dico autem non esse le simpliciter: et enim simpliciter ente bonum melius -, duorum igitur alterum: si non ens est nullatenus ens, multo magis neque malum est, quod et nullatenus ente amenenoteron (id est immansivius), ut pronuntiavit sermo; magis enim a bono distat malum quam non ens. Significant autem qui le non esse ante male esse ponunt, <quibus > quod autem ab illo remotius anoysion (id est sine essentia) magis proquinquiori; ipsum ergo le nullatenus ens est magis quam dictum malum: multo ergo magis omniquaque non ente non est le malum ${ }^{6}$.

Che bisogno c'è di molti discorsi? Infatti, se l'uno e ciò che diciamo la natura del bene è al di là dell'essere, allora il male è al di là del non-essere in sé - intendo [qui] il non-essere in senso assoluto, perché il bene [scil. il bene primo] è superiore all'essente in senso assoluto -; delle due l'una: <...>>; se [invece] il non-essente [in questione] è assolutamente non essente [scil. è il non-essente assoluto], tanto più, allora, neppure il male è essente ${ }^{8}$, poiché questo [scil. il male] è ancor più inconsistente del non-essente assoluto, come ha mostrato il discorso; il male, infatti, è distante dal bene [primo] più di quanto sia distante da quest'ultimo il non-essente. Mostrano [ciò] coloro che pongono il non-essere prima dell'essere malamente ${ }^{9}$; ciò che è più lontano da

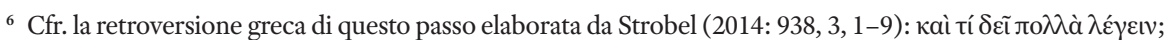

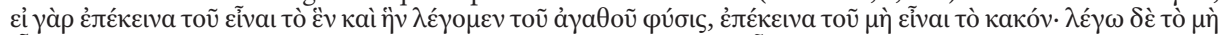

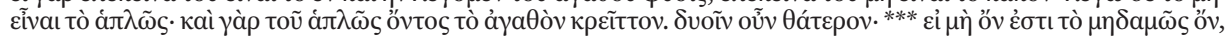

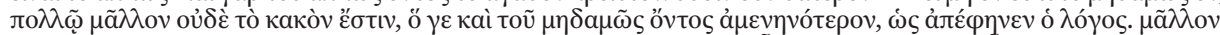

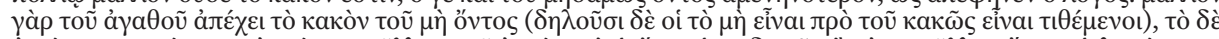

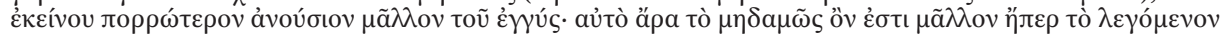

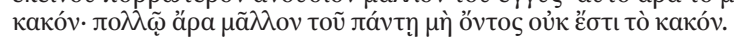

7 Opsomer e Steel (2003: 58, 133) sostengono, a mio avviso a ragione, che in questo passo, dopo alterum, sia andato perduto un segmento testuale che doveva contenere l'esposizione di una delle due posizioni alternative introdotte dall'espressione duorum igitur alterum, precisamente la prima ipotesi dell'alternativa, ipotesi che viene negata come impossibile per confermare la seconda ipotesi, che nel testo tràdito è esposta immediatamente dopo alterum. In linea con un precedente studio di Steel (1997: 98, nota 17), i due studiosi propongono di colmare la lacuna sulla base di un passo parallelo dello Pseudo-Dionigi Areopagita, con la seguente integrazione: <aut non ens est nullatenus ens aut quod superessentiale; sed impossibile le malum esse ultra non esse superessentiale, quod bonum est; > (cfr. Opsomer, Steel 2003: 133, rif. a 3, 4), che traducono: «<Non-being is either absolutely-notbeing or what is beyond being. But it is impossible that evil is beyond superessential non-being, which is the good. >» (ivi: 58). Il passo pseudo-dionisiano in questione è DN IV 19, $716 \mathrm{C}-\mathrm{D}$, vol. I, 163, 21-23 Suchla: tò

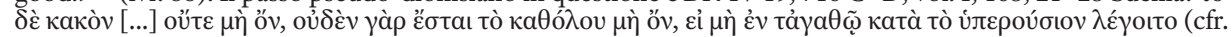
Steel 1997: 97-99). Per la segnalazione di una lacuna dopo alterum, cfr. anche Strobel (2014: 702-703, 3, 4); Isaac (2003b: loc. cit.) e Boese (1960: 176), invece, non segnalano alcuna lacuna.

${ }^{8}$ Si potrebbe anche rendere il passo in questo modo: «se il non-essente [in questione] è assolutamente non esistente, tanto più allora neppure il male esiste».

9 Alla linea 10, dopo ponunt provo a tralasciare l'integrazione < quibus> Proposta da Isaac (2003b: loc. cit.). Anche Boese (1960: 176, 3.8) indica una lacuna, ma non propone integrazioni. Per altro verso, Westerink (1962: 
quello [scil. dal bene], dunque, è più insussistente di ciò che è più vicino [al bene]; il non-essente assoluto stesso, quindi, è più essente [scil. esistente] di ciò che è detto "male": ne consegue che il male è molto più non essente [scil. non esistente] del non-essente assoluto (Procl. DMS 3 , $1-15$, trad. mia).

Secondo la corrispondenza inversa del male al bene primo (qui indicato come "la natura del bene", la quale, nella sua identità con il semplicemente uno, è il principio primo di tutte le $\cos \mathrm{e}^{10}$ ), posto che il bene primo è al di là dell'essente in senso assoluto (simpliciter ens, tò á $\pi \lambda \tilde{\omega} \varsigma$ ô $v^{11}$, che qui dovrebbe indicare la realtà ontica nel complesso dei suoi ordinamenti), allora il male si configura, in modo simmetrico, come al di là del non-esse-

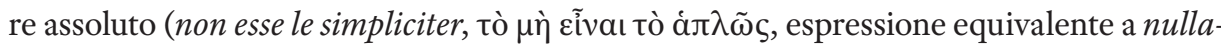
tenus ens, tò $\mu \eta \delta a \mu \tilde{\omega} \varsigma$ oै $v$, che ricorre poco dopo nel testo sopra citato) ${ }^{12}$. In questo argomento, il dato dell'identificazione del non-essente "trasceso" dal male con il non-essente assunto nella sua assolutezza, cioè con il nulla, è stabilito sulla base della corrispondenza tra l'essente trasceso dal bene primo e il non-essente trasceso dal male. Se il bene primo, infatti, è al di là dell'essente assunto nella sua assolutezza, allora il male, come termine concettualmente antitetico al bene primo, è al di là del non-essente assunto anch'esso, a suo modo, in senso assoluto, il quale si configura come il termine concettualmente antitetico all'essente in senso assoluto. Come vedremo, in questi due casi tra i quali è istituita una corrispondenza, si delineano due modi diametralmente opposti di configurare la "trascendenza". Il male, dunque, così concepito, è posto come ancor più inesistente del non-essente assoluto ${ }^{13}$ proprio perché è messo in rapporto inverso con il bene primo e non con un qualche bene determinato. Con ciò si comprende che il male di cui si sta parlando, ancorché sia presentato in modo generico, vada assunto come assoluto, così come assoluto è il bene al quale esso è messo in relazione, e assoluti - ciascuno a suo modo - sono i due termini che sono "trascesi", rispettivamente, dal bene primo e dal male.

Per quanto concerne l'equivalenza stabilita da Proclo, riguardo al male, tra l'essere “al di là anche del non-essente assoluto" e l'essere "ancor più inesistente del non-essente assoluto", possiamo notare che la maggiore inesistenza - o, potremmo dire, l'inesistenza a maggior titolo - del male (in sé) rispetto al non-essente assoluto è anche espressa con

$166)$ in questo passo non segnala alcuna lacuna. In quest'ultima direzione cfr. Opsomer, Steel (2003: 58-59 e 133, rif. a $3,6-8)$.

10 Sull'identità dell'uno e del bene, quali termini indicanti entrambi il principio primo di tutte le cose, cfr. per es. Procl. in Prm. VI 1097, 8-16; VII 511, 12-20 Steel et al.; Procl. ET prop. 13, 14, 24-16, 8 Dodds; Procl. Theol.Plat. II 6, 40, 1-41, 17. Su quest'identità nel platonismo tardo-antico cfr. Steel (1989).

${ }_{11}$ Per le retroversioni greche delle espressioni latine dell'opusculum procliano, cfr. Strobel (2014).

12 Si può anche notare che nel passo procliano in questione, Erler (1978: 12), relativamente all'espresione ultra ipsum non esse malum (riguardo alla quale l'inciso dico autem non esse le simpliciter intende chiarire

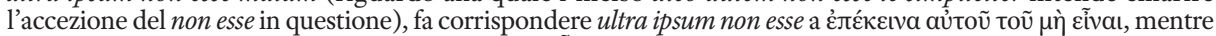

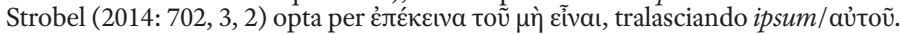

13 In Procl. DMS 3, 7 il termine amenenoteron (ả $\mu \varepsilon v \eta v o ́ t \varepsilon \rho o v)$ manifesta, in ultima istanza, il significato iperbolico di "più inesistente". 
l'assunto secondo cui la lontananza del male (in sé) dal bene primo è maggiore di quella che separa da quest'ultimo il non-essente assoluto. In questo caso, la maggiore distanza - metafisicamente intesa - di un termine dal bene primo si traduce, in una prospettiva generale, in inferiorità sul piano gerarchico. Infatti, mentre il non-essente assoluto è negazione all'essente, invece il male assoluto è negazione del bene, e il bene è metafisicamente anteriore all'essente. Secondo Proclo, l'assunto secondo cui il male è distante dal bene ancor più di quanto sia distante da quest'ultimo il non-essente assoluto, è un dato che è anche attestato, su un piano che vorrei definire esistenziale e valoriale, da coloro che

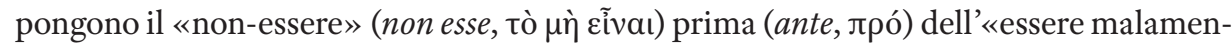

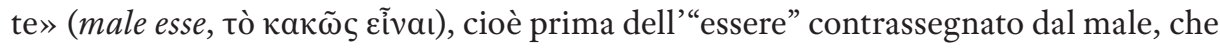
qui vorrei rendere con l'espressione «male-essere». Come è possibile chiarire sulla base di altri testi procliani, il filosofo allude qui a coloro che preferiscono la morte a una vita segnata dalla viltà o dalla miseria. Proclo rintraccia una formulazione esemplare di questo assunto nell'Alcibiade primo, sia nel passo in cui Socrate mette in evidenza il fatto che Alcibiade preferirebbe morire subito, piuttosto che vivere con ciò che possiede senza poterlo incrementare ( $A l c .1105 \mathrm{a}$ ), sia nel passo in cui Alcibiade, nel rispondere a Socrate, che gli chiede a quale prezzo sarebbe disposto a essere privato del coraggio, dichiara che, se dovesse essere vile, non accetterebbe neppure di vivere $(A l c .1115 \mathrm{~d})^{14}$. In quest'ottica, sul piano di una scala di valori sperimentabile nella vita vissuta, si delinea una conferma di un principio generale che trova la sua piena giustificazione sul piano metafisico, guardando al differente statuto del non-essere e del male-essere: il non-essere, come Proclo spiega altrove, è migliore del male-essere perché, mentre il non-essere è privazione dell'essente, invece il male-essere è privazione del bene ${ }^{15}$, e il bene costituisce un orizzonte metafisico di livello superiore a quello dell'essente. Così, in questo contesto, sul piano delle corrispondenze da un lato tra il male-essere di una vita squallida e il male in sé, e dall'altro lato tra il non-essere dell'assenza di vita e il non-essente assoluto, si può rilevare che anche nell'ambito dell'esperienza vissuta, in cui il male-essere si presenta come peggiore del non-essere e quest'ultimo è preferibile a quello (cioè è posto "prima"

${ }^{14}$ Cfr. rispettivamente Procl. in Alc. I 144, 4-7 (nell'ambito del commento a Pl. Alc. 1 105a Burnet) e II 337, 12-14 (nell'ambito del commento a Pl. Alc. 1 115c) Segonds. Si veda inoltre Procl. in Rmp. II 89, 24-28 Kroll, in cui il non-essere è presentato come un male minore del male-essere.

15 Procl. DMS 39, 57-59. In questo contesto Proclo afferma che un male che corrompe la potenza di una cosa è maggiore di un male che corrompe la sostanza di un'altra cosa nel caso in cui la potenza della cosa in questione sia superiore alla sostanza dell'altra cosa, come nel caso delle potenze dell'anima rispetto alla sostanza corporea. Egli identifica questo male superiore con il male "assolutamente terribile" (omnino dira malitia)

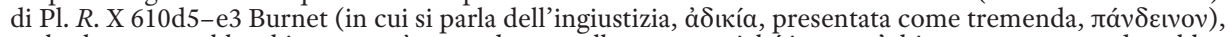
male che non sarebbe chiamato così se conducesse alla morte, poiché in quest'ultimo caso esso condurrebbe rapidamente al non-essere le anime che ne sono affette e, appunto, il non-essere è migliore del male-essere. Con ciò egli intende mostrare che il male delle anime è maggiore di quello dei corpi, perché mentre quest'ultimo, aumentando, ha il suo compimento nel non-essere, cioè nella morte fisica, la quale comporta la fine di questo stesso male, invece il primo ha il suo compimento nel male-essere, cioè in un'esistenza segnata dal male (cfr. Procl. DMS 39, part. 44-62). 
del male-essere), trova conferma il fatto che il male (in sé) è ancor più lontano dal bene primo di quanto non lo sia il non-essente assoluto ${ }^{16}$.

Ora, sul piano metafisico, la tesi procliana secondo cui il male (in sé) è al di là anche del non-essente assoluto va inquadrata come un rilievo basilare della formulazione di un argomento con cui Proclo intende dimostrare l'impossibilità dell'esistenza del male in $\mathrm{sé}^{17}$. Si tratta di un argomento a fortiori. Il Licio, infatti, nello sviluppare la sua tesi, sostiene che, posto che il non-essente assoluto non esiste in alcun modo, allora si deve affer-

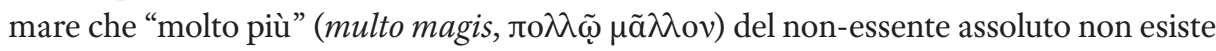
il male in sé, dato che quest'ultimo si delinea come ancor più inconsistente/inesistente del non-essente assoluto, ovverosia - secondo quanto egli ha prospettato poco prima - come al di là anche del non-essente assoluto. In questo ragionamento, l'espressione multo magis, che modula i termini del confronto tra il non-essente assoluto e il male in sé, nella trama logico-argomentativa in cui è inserita, può essere debitamente resa, ancor meglio che con "ancor più", con "a maggior ragione". Ciò rende manifesto il fatto che in questo argomento il male in sé, dal punto di vista metafisico, è fattualmente dichiarato non esistente "al pari” del non-essente assoluto, ovverosia è ridotto al nulla, in forza di un ragionamento che, nella sua forma radicale, intende non lasciare alcuno spazio speculativo di manovra per la postulazione di una qualche forma di esistenza del male in sé. Inquadrato come "al di là anche del nulla" e come "più inesistente perfino del nulla", il male assoluto è presentato, per così dire, come ancor più nulla del nulla $\mathrm{o}$, potremmo dire, come nulla a maggior titolo del nulla. Questo assunto, però, va letto sulla base del rilievo secondo cui, nel pensiero procliano, assolutamente nulla può essere ancor più nulla del nulla, ovverosia ancor più inesistente di ciò che per definizione è posto come assolutamente inesistente, in una visione in cui non si può porre alcuna distinzione tra modi o gradi del nulla, che è assunto come una nozione rigorosamente univoca. Il male in sé, allora, posto come "al di là anche del non-essente assoluto", in una certa ottica si presta a essere dichiarato non esistente "a maggior titolo" del non-essente assoluto, ma di fatto ciò equivale a dichiararlo non esistente "al pari” del non-essente assoluto. Ciò in una

${ }^{16}$ A questo riguardo, si può notare che nei casi tratti dall'Alcibiade primo il male-essere non si presenta come "più inesistente" del non-essere. Il male-essere, infatti, consistendo in una vita segnata dal male inteso come miseria e assenza di virtù, si dà nell'orizzonte dell'esistenza, in una prospettiva in cui il male in questione, a differenza del male puro, è assunto come privazione parziale di bene in un essere vivente. Il male-essere, in questo caso, si rivela peggiore del non-essere (qui la morte) proprio in quanto, come male-essere, si dà nell'essere e si presenta come preferibile al non-essere. Potremmo dire che la maggiore lontananza del male dal bene rispetto a quella che separa da quest'ultimo il non-essente assoluto, nel caso dell'esperienza vissuta, si manifesta su un piano etico-valoriale, in cui appunto il non-essere è preferibile al male-essere.

${ }_{17}$ Mi sembra interessante segnalare la lettura di O'Brien (2017: part. 155-156 e nota 55), il quale, nell'ambito di una stimolante ricognizione di aspetti salienti della discettazione procliana sul male in $D M S$ 9, a proposito della presentazione dell' "ultimate evil" come "beyond what is not in any way at all (nullatenus entis ultra), scrive: «In asserting that ultimately evil lies "beyond what is not in any way at all" (Procl. DMS 9.6-7), is Proclus perhaps more concerned to emphasize the utter unreality of ultimate evil than to present his reader with the baffling concept of a negation more ultimate even than the denial of any partecipation at all in being? Very possibly so. But that is what his argument has led him to» (O’Brien 2017: 156), e nella nota 55 (ibid.) aggiunge: «"Very possibly": I originally wrote "possibly". An anonymous reviewer urged me to change this to "certainly". [...]». Secondo la mia comprensione della questione, in questo caso propendo decisamente per "certainly", in linea con l'anonimo reviewer del sopra citato saggio di O'Brien. 
visione in cui il male così assunto, cioè come male assoluto, coincide con il non-essente assoluto, pur potendo essere distinto da quest'ultimo secondo la nozione e pur potendo aprire, rispetto a esso, uno spazio di riflessione, sul piano - potremmo dire - del "valore", in ambito tanto metafisico quanto etico. L'espressione "al di là anche del non-essente assoluto", così, si presenta come una formula iperbolica ed enfatica, votata a sancire in modo incontrovertibile la non esistenza del male in sé. Lo stesso vale per la formula a essa equivalente, "più inconsistente (cioè più inesistente) anche del non-essente assoluto" (et

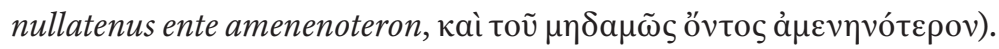

A questo riguardo, è utile spendere qualche parola sul valore che la preposizione

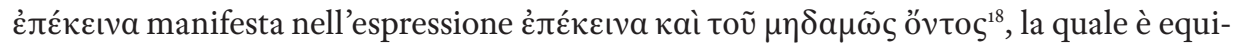
valente all'espressione latina ultra ipsum non esse - dico autem non esse le simpliciter ${ }^{19} \mathrm{e}$, come vedremo, corrisponde all'espressione nullatenus entis ultra che ricorre in seguito nel medesimo opusculum (cfr. Procl. DMS 9, 9.). Nella suddetta espressione la preposi-

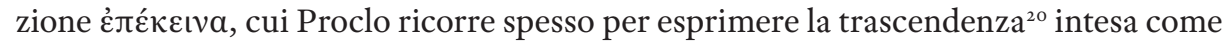
cifra di superiorità metafisica di qualcosa rispetto a qualcos'altro, è usata invece, in una prospettiva capovolta, per indicare uno stare "dall'altra parte" che è orientato non verso l'alto, bensì verso il basso, dunque un porsi "al di sotto" e non "al di sopra” di qualcosa, potremmo dire una sorta di trascendenza rovesciata, indice metafisico d'inferiorità e non

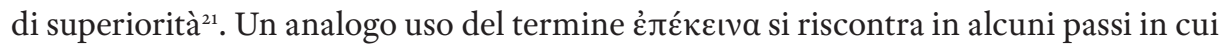
Proclo prospetta l'idea che il non-essente assoluto ( - "al di sotto" - degli ordini infimi della realtà, in un'ottica in cui esso è assunto nella sua mera non esistenza ${ }^{22}$.

In un passo del De malorum subsistentia Proclo presenta il non-essente assoluto - che

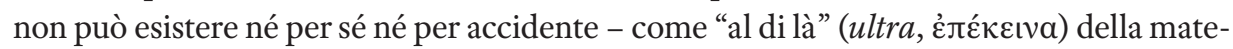
ria, vista come la "natura ultima“, cioè come il più basso ordine del reale ${ }^{23}$ :

Et le non ens ipsum, quod quidem nullatenus ens aliud et ultra ultimam naturam, que secundum accidens est, neque secundum se neque secundum accidens esse potens ${ }^{24}$.

${ }_{18}$ Cfr. Procl. in Ti. I 374, 14-15; Procl. Theol.Plat. I 18, 86, 20-21.

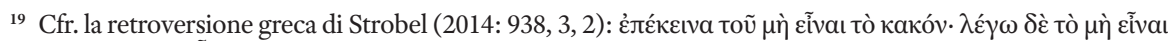

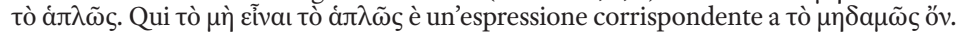

${ }^{20}$ Sul lessico protologico della trascendenza in Proclo cfr. Beierwaltes (2014: 352-353 e nt. 65).

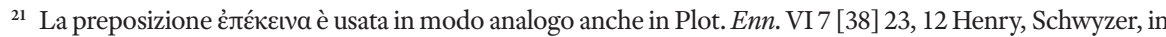

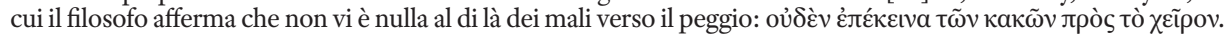

22 Questi passi, che riporto di seguito, sono menzionati da Moutsopoulos (2006: 148, noti 30-31), il quale, a questo riguardo, parla di una «transcendance inversée, "négative" propria del «non être qui transcende les divinités», presentandola come «assez énigmatique» (con riferimento a Procl. in Ti. I 209, 26). Tale carattere enigmatico, a mio avviso, si risolve alla luce del fatto che questa "trascendenza" indica una relazione puramente concettuale, e il non-essente che è "al di là" degli dèi si rivela un termine che coincide con il nulla.

${ }^{23}$ Per questa concezione della materia cfr. anche Procl. Theol.Plat. I 12, 57, 18.

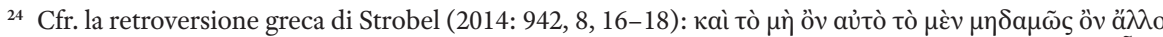

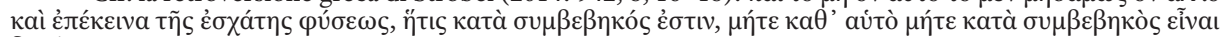

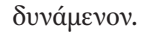


$\mathrm{E}$ [per quanto riguarda] il non-essente stesso, vi è il non-essente che non è altro che non-essente assoluto e che è al di là della natura ultima, la quale è essente per accidente, [il non-essente] che non può essere né per sé né per accidente (Procl. DMS 8, 26-29, trad. mia).

Anche nel Commentario sul "Timeo" il filosofo afferma che il non-essente assoluto è "al di là" della materia, la quale, come ultimo ordine ipostatico, costituisce il limite infimo della totalità dell'esistente ed è anch'essa presidiata e ordinata dagli dèi; "al di là"

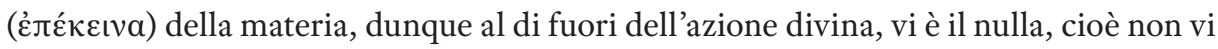
è niente:

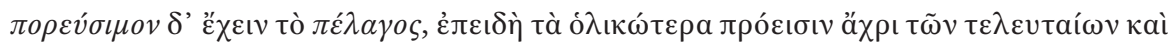

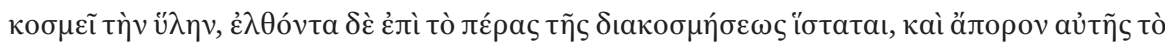

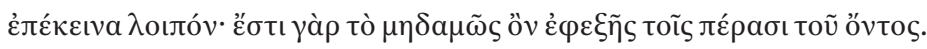

Il "mare" è "navigabile" poiché i generi [divini] più universali procedono fino alle realtà ultime e conferiscono ordine alla materia, e giunti al limite [infimo] dell'ordinamento [dell'intera realtà] essi si fermano, e ciò che resta al di là di questo [ordinamento] è inaccessibile; infatti, fuori dai limiti dell'essente [scil. dell'esistente] vi è il non-essente assoluto (Procl. in Ti. I 178, 26-31, trad. mia) ${ }^{25}$.

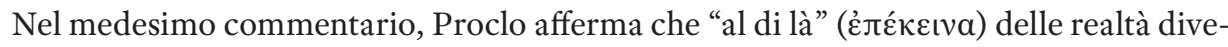
nienti e corruttibili, viste come le realtà infime, vi è il non-essente assoluto, cioè non vi è niente:

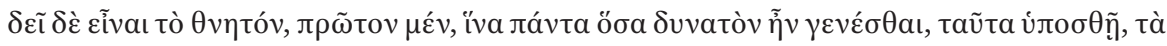

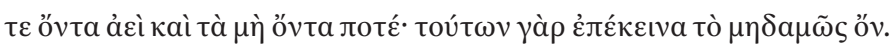

Ma vi deve anche essere ciò che è mortale, in primo luogo affinché tutte quante le cose che potevano venire all'essere esistessero [effettivamente], sia quelle che sono sempre, sia quelle che talvolta non sono; infatti, al di là di queste ultime vi è il non-essente assoluto (Procl. in Ti. III 222, 28-31, trad. mia).

\section{E ancora:}

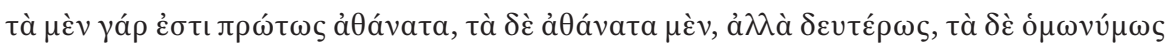

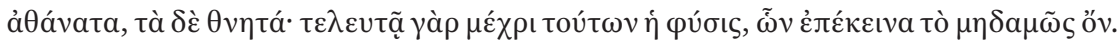

Infatti, mentre alcune cose sono primariamente immortali, altre sono immortali, ma in modo secondario, altre sono immortali per omonimia, altre invece sono mortali: perché la natura

${ }_{25}$ Proclo qui avanza un'interpretazione di Pl. Ti. 24e4-5 Burnet. 
si conclude con queste ultime, al di là delle quali vi è il non-essente assoluto (Procl. in Ti. III $231,20-23$, trad. mia).

Si può anche citare un altro passo del Commentario sul "Timeo", in cui Proclo, con riferimento all'assunto della presenza degli dèi in ogni ordine del reale, dunque anche nelle

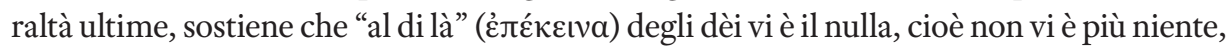
nel senso che non vi è nulla che si ponga fuori dalla loro azione e che si sottragga alla loro funzione fondativa:

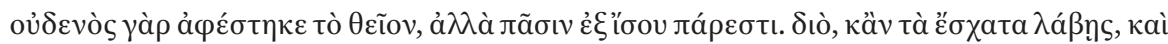

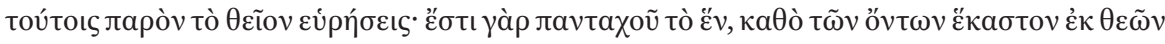
ن่

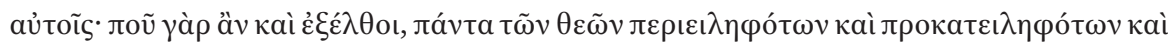

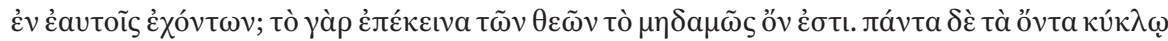

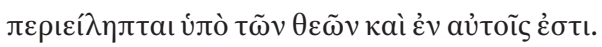

infatti il divino non è separato da alcuna cosa, ma è ugualmente presente in tutte le cose. Perciò, anche se tu prendessi in considerazione le realtà ultime, anche in queste troveresti la presenza del divino; infatti l'uno è dovunque, nella misura in cui ciascuna delle cose ha ricevuto l'esistenza dagli dèi, ed esse, procedendo tutte quante dagli dèi, non si sono poste fuori da questi, bensì sono radicate in essi. In che modo, infatti, potrebbero essersi poste fuori [dagli dèi], dato che gli dèi abbracciano tutte le cose, le afferrano preventivamente e le tengono in se stessi? Perché ciò che è al di là degli dèi è il non-essente assoluto. Tutti gli essenti sono circondati dagli dèi e sono in essi (Procl. in Ti. I 209, 19-28, trad. mia).

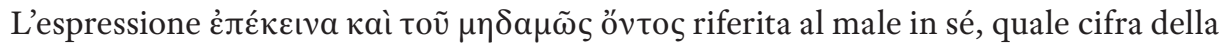
manifesta impossibilità dell'esistenza del male concepito come del tutto separato dal bene, ricorre anche in un passo del Commentario sul "Timeo", in cui essa manifesta il proprio profilo speculativo nelle sue istanze e implicazioni. In questo caso, va notato

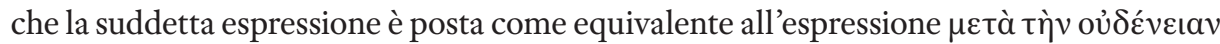

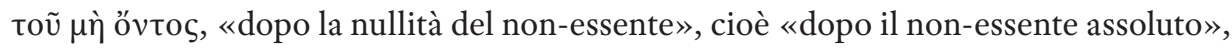

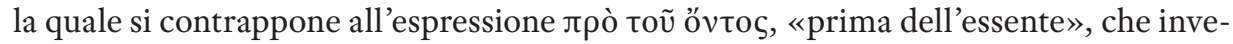

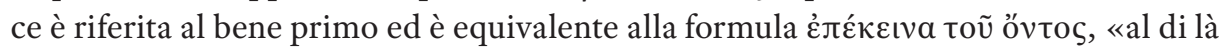
dell'essente»:

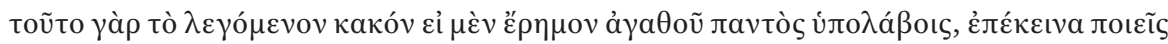

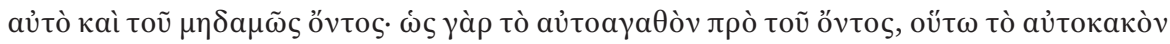

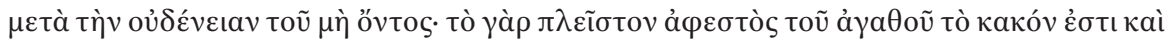

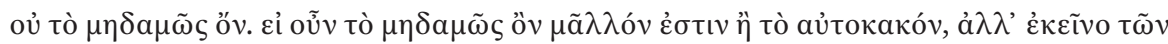

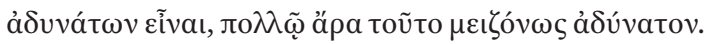


Se tu assumessi ciò che è detto "male" come separato da ogni bene, allora lo renderesti al di là anche del non-essente assoluto: perché, come il bene in sé è anteriore all'essente, così il male in sé è posteriore alla nullità del non-essente ${ }^{26}$; infatti, ciò che più si è allontanato dal bene è il male [in sé] e non il non-essente assoluto. Se dunque il non-essente assoluto esiste più del male in sé, e se quello [scil. il non-essente assoluto] rientra tra le cose impossibili, allora tanto più questo [scil. il male in sé] è impossibile [oppure: allora questo è tanto più impossibile (del non-essente assoluto)] (Procl. in Ti. I 374, 13-20, trad. mia) ${ }^{27}$.

Porre il male come separato da ogni bene, cioè come male in sé, significa quindi porlo come al di là del nulla, e ciò mostra l'impossibilità dell'esistenza del male così concepito. Con riferimento al non-essente assoluto, nel passo sopra citato la preposizione

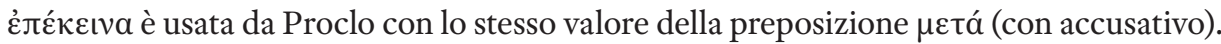
La preposizione $\mu \varepsilon \tau a ́$ (con accusativo), in generale, è indice di "posteriorità" metafisicamente intesa come inferiorità e subordinazione di un ordine rispetto a un altro che, nella scala del reale, si pone come superiore e lo trascende. Tale preposizione si contrappone alla preposizione $\pi \rho$ ó, la quale, invece, è indice di "anteriorità" metafisicamente intesa come superiorità di un ordine rispetto a un altro che, nella scala del reale, si pone come inferiore. Come tale, la preposizione лрó appartiene al lessico protologico procliano della trascendenza ${ }^{28}$. Se quindi il bene primo è "anteriore" all'essente assunto nella sua assolutezza, nel senso che è al di sopra di esso e ne costituisce il principio, il male in sé, in modo inverso, si configura come "posteriore" al non-essente assoluto, nel senso che è "al di sotto" di questo e va concepito come ancor "più lontano" dal bene primo di quanto non lo sia il non-essente assoluto. Così, il non-essente assoluto è concepito, in termini paradossali, come più esistente del male in sé, e quest'ultimo come perfino più inesistente del non-essente assoluto.

Su questa linea, posto che il non-essente assoluto fa parte delle cose impossibili (cioè che non possono in alcun modo esistere), ovverosia è la cosa impossibile per eccellenza, allora il male in sé, che è al di là anche del non-essente assoluto, rientra tra le cose impossibili ancor più e a maggior ragione del non-essente assoluto $\mathrm{o}$, se vogliamo, è molto più impossibile dell'impossibile non-essente assoluto. Alla luce di questo rilievo, andrebbe rilevato che, riguardo al male puro, la fattuale equivalenza tra "l'ancor più inesistente del non-essente assoluto" e "il non-essente assoluto", si traduce nell'equivalenza tra "l'ancor più impossibile dell'impossibile" (il male puro) e "l’impossibile" (qui il non-essente

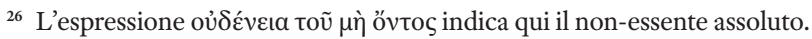

27 Festugière (2006: 235 e nota 3), sulla base delle emendazioni proposte - «En 374.18 lire $\mu \tilde{a} \lambda \lambda$ ov ع́ $\sigma \tau \iota$

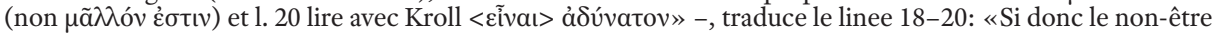
absolu a plus d'existence que le Mal-en-soi, et si d'autre part ce non-être absolu est du nombre des choses qui ne peuvent exister, bien plus encore le Mal-en-soi sera-t-il une chose qui ne peut exister». In ogni caso, qui l' "essere impossibile" coincide con il "non potere esistere".

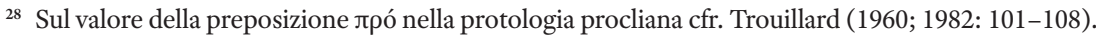


assoluto ${ }^{29}$. Proprio come il mero non esistente, infatti, anche l'impossibile, stricto sensu, non ammette gradazioni interne; ne consegue che, posto il non-essente assoluto come impossibile, non può darsi qualcosa che, rispetto a esso, sia in senso proprio ancor più impossibile ${ }^{30}$. L'argomento procliano, quindi, intende mostrare l'assunto secondo cui, posto che il nulla è impossibile, allora il male in sé, che si configura come "al di là" del nulla, a fortiori è anch'esso impossibile e, come il nulla, in nessun modo può essere posto come esistente. Il male in sé, insomma, è identico al nulla. Delineati tali assunti, nella prosecuzione del testo in questione del Commentario sul "Timeo" Proclo avanza la tesi, sviluppata nel De malorum subsistentia, dell'esistenza del male mescolato al bene e, dunque, anch'esso buono, in quanto esistente (cfr. Procl. in Ti. I 374, 20-28).

In sintesi, l'argomento procliano incentrato sulla corrispondenza inversa tra il bene assoluto e il male assoluto, è mirato non a prospettare una qualche modalità infima di esistenza del male assoluto in opposizione alla suprema esistenza del bene primo, bensì a sancire la non esistenza del male assoluto, sulla base del suo stesso carattere di assolutezza che si delinea nel confronto con il bene primo ${ }^{31}$. Tale corrispondenza, infatti, non riguarda due termini entrambi esistenti, in quanto all'assoluta sovraessenzialità del bene primo, che nel pensiero procliano rappresenta il più alto rango di esistenza, corrisponde l'assoluta inessenzialità - cioè la mera non esistenza - del male in sé ${ }^{32}$. Con riferimento a questa differenza di fondo, nella delineazione procliana della corrispondenza tra il bene primo e il male puro, si prospetta la contrapposizione tra due forme radicali di "trascendenza", le quali, in modi diversi, non lasciano spazio alla postulazione di termini ulteriormente "trascendenti" rispetto a quelli posti. Per un verso, la trascendenza del bene primo rispetto all'essere in sé è cifra dell'esclusiva modalità di esistenza del principio primo, al di sopra del quale non vi è assolutamente più nulla ${ }^{33}$. In questo caso siamo al cospetto del principio la cui esistenza, nell'ambito dell'esistente, si configura come la forma originaria e suprema di “esistenza”, inesprimibile e differente da ogni altra tipologia e modalità di esistenza. Per altro verso, la trascendenza del male in sé rispetto al non-essente asso-

\footnotetext{
29 Sull'impossibilità del non-essente assoluto cfr. Procl. in Prm. V 1000, 6-7, in cui il $\mu \eta \delta a \mu \tilde{\omega} \varsigma$ öv

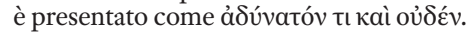

30 Proclo altrove (cfr. Theol.Plat. I 10, 41, 13-20) ricorre a un'espressione iperbolica per indicare le conclusioni assurde cui giungono, secondo la sua interpretazione, le ultime quattro ipotesi del Parmenide, vuote e impossibili, basate sulla supposizione della non esistenza dell'uno (il semplicemente uno); secondo lo Scolarca, queste ipotesi conducono alle conseguenze, per così dire, più impossibili tra le impossibili (cfr. Theol.Plat. I 10, 41,

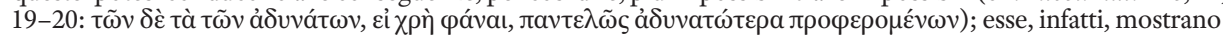
che, se l'uno non esistesse, non esisterebbe assolutamente nulla (cfr. Procl. Theol.Plat. I 10, 41, 7-20 e I 12, 58, 8-22; Procl. in Prm. VI 1056, 1-1057, 4).

${ }^{31}$ Sul rifiuto procliano dell'argomento per analogia volto ad affermare l'esistenza del male in sé in corrispondenza dell'esistenza del bene primo cfr. Fiorentino (1998: 74-75).

${ }^{32} \mathrm{Al}$ di là del raffronto tra il bene primo (quale termine esistente) e il male assoluto (quale termine non esistente), sul versante del male, il porsi del male in sé come "al di là" del non-essente assoluto, esprime su un piano concettuale un rapporto tra due termini non esistenti, a differenza di quanto si registra sul versante del bene, in cui il porsi del bene primo come "al di là" dell'essente in sé esprime un rapporto tra due termini esistenti.

${ }_{33}$ Sull'impossibilità dell'esistenza di qualcosa al di là dell'uno in sé (cioè di una causa dell'uno in sé) cfr. Procl. in Prm. VII 1143, 27-1144, 31; Procl. ET. prop. 5, 6, 16-20 e prop. 12, 14, 1-23; Procl. Theol.Plat. II 1, 12, 25-14, 11.
} 
luto sancisce la non esistenza di un termine che, al pari di ciò che esso "trascende", non può che essere posto come pura vacuità anipostatica.

Vediamo meglio come si configura il rapporto tra il male in sé e il non-essente assoluto in questo scenario speculativo procliano.

\section{Il male in sé e il non-essente assoluto}

Nel $\$ 9$ del De malorum subsistentia Proclo presenta nuovamente il male come "al di là anche del non-essente assoluto", con specifico riferimento al male in sé, che adesso viene esplicitamente distinto dal male commisto al bene. Questo assunto, alla luce delle considerazioni prospettate nei $\$ \$ \$ 6-8$, è integrato nella trama argomentativa della tesi dell'esistenza del male, la quale trova la sua esatta definizione nella postulazione dell'esistenza del male commisto al bene. A configurarsi come al di là anche del non-essente assoluto, quindi, è il male in sé e non il male relativo, il quale invece, a suo modo, fa parte delle cose esistenti.

La distinzione tra il male in sé e il male mescolato al bene, per certi versi accostabile a quella tra il bene in sé e il bene commisto ad altro, è delineata con uno specifico riferimento alla distinzione tra il non-essente assoluto e il non-essente relativo. Infatti, così come, per un verso, bisogna porre la distinzione tra il non-essente assoluto, che non esiste in alcun modo, e il non-essente relativo, che va annoverato tra le cose esistenti, per altro verso, in modo analogo, bisogna anche porre la distinzione tra il male assoluto, il quale, come il non-essente assoluto, non esiste in alcun modo, e il male commisto al bene, il quale, come il non-essente relativo, fa parte delle cose esistenti ${ }^{34}$. Il male assoluto non esiste perché è assunto come totalmente privo del bene e quindi anche dell'essere, dato che l'essere non può sussistere senza il bene; il male commisto al bene, invece, è esistente in virtù della sua partecipazione del bene, la quale comporta la sua appartenenza al piano dell'essere:

Sicut igitur, si le non ens quereret quis utrum est aut non est, quod quidem omniquaque non ens, nullatenus esse participans, diceremus nullatenus ens; hoc autem modo quodam non ens in entibus connumerare concederemus hec amanti; secundum eadem utique et malum - quoniam et hoc duplex, hoc quidem solummodo malum, hoc autem non impermixtum ad bonum - hoc quidem videlicet nullatenus entis ultra ponemus, quanto bonum entis ultra, hoc autem in entibus ordinabimus; neque enim ab esse propter boni mesiteiam (id est mediationem) neque a bono propter esse potens adhuc manere desertum: simul enim ens est et bonum. Et quod quidem omniquaque malum primi bonorum decidentia ens et velut exitio,

${ }^{34}$ Su ciò cfr. Maurette (2004: 147). 
merito et ente privatum est: quid enim utique in entia progressum habebit, bono participare non potens? ${ }^{35}$

Così, dunque, se qualcuno domandasse se il non-essente sia o non sia, diremo che ciò che è in ogni modo non-essente, in quanto non partecipa in alcun modo dell'essere, è assolutamente non-essente [scil. non esistente]; invece, a chi domanda ${ }^{36}$ ciò, concederemo di annoverare tra gli essenti [scil. tra le cose esistenti] il non-essente che è tale secondo un certo rapporto. Ugualmente, anche nel caso del male - poiché anche questo è duplice: quello che è solamente male e quello che, invece, non è non mescolato al bene - porremo il primo [scil. il male che è solamente male] al di là del non-essente assoluto nella stessa misura in cui il bene [scil. il principio primo] è al di là dell'essente, e il secondo [scil. il male che non è non mescolato al bene] tra gli essenti, perché quest'ultimo non può restare privo né dell'essere, per via della mediazione del bene, né del bene, per via dell'essere: infatti [questo male] è insieme essente e buono. E il male assoluto, essendo una caduta e come un'uscita dal primo dei beni, a ragione è privo anche dell'essere: infatti, come potrebbe entrare nel novero degli essenti, non potendo partecipare del bene? (Procl. DMS 9, 1-17, trad. mia).

Proclo, così, stabilisce una corrispondenza, da un lato, tra il non-essente assoluto e il male in sé, come termini entrambi non esistenti, e, dall'altro lato, tra il non-essente relativo e il male commisto al bene, come termini entrambi annoverabili tra le cose esistenti. Egli stabilisce questa duplice corrispondenza sulla scia di prospettive speculative tracciate nel precedente $\$ 8$ dell'opusculum, in cui, con riferimento alle nozioni basilari di male, bene, essente e non-essente, delinea un sistema di distinzioni tra determinazioni contrapposte, che provo a schematizzare nel seguente modo: [il male (a)] il male puro, non commisto al bene (a1), contrapposto al male non puro, mescolato al bene (a2); [il bene (b)] il bene primo, che non è nient'altro che bene (b1), contrapposto al bene commisto ad altro (b2); quest'ultimo tipo di bene è a sua volta diviso nel bene non mescolato alla privazione (b2.1) e nel bene mescolato alla privazione (b2.2); [l'essente (c)] il realmente essente, solamente essente (c1), che è posto al livello superiore (rispetto all'essente mescolato al non-essente), contrapposto all'essente che è mescolato al non-essente e che è individuato negli ultimi ordini degli essenti (c2); [il non-essente (d)] il non-essente assoluto (d1), cioè il nulla, contrapposto al non-essente che è congiunto all'essente (d2); quest'ultimo tipo di non-essente è articolato in privazione dell'essente $(\mathrm{d} 2.1)$ e in alterità $(\mathrm{d} 2.2)$, le

35 Cfr. la retroversione greca di questo passo elaborata da Strobel (2014: 942-943, 9, 1-10):

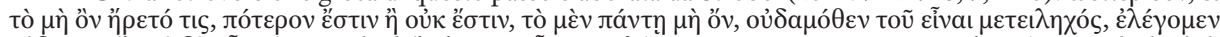

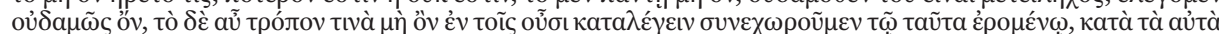

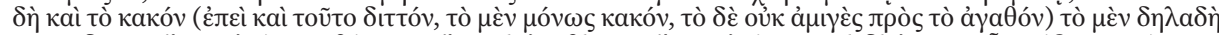

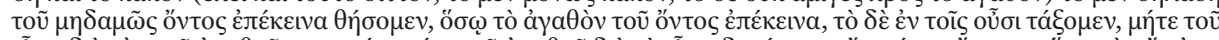

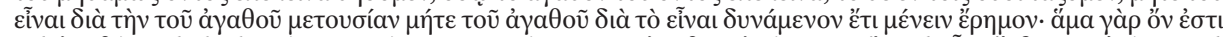

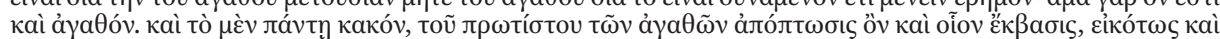

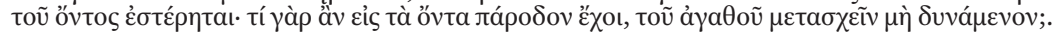

${ }^{36}$ Qui Guglielmo di Moerbeke traduce erroneamente con amanti il greco épouév $\omega$; cfr. Strobel (2014: 734, $9,4$ e $943,9,3)$. 
quali sono disposte su due distinti piani gerarchici (cfr. Procl. $D M S$ 8, 8-38). Si delinea, così, un articolato quadro concettuale in cui si stagliano dieci nozioni, le cui connotazioni, contrapposizioni e interrelazioni concorrono ad affrescare una mappatura tipologica di diversi ordini ipostatici, modi di esistenza e concetti limite di termini inesistenti. Mi limito qui a rilevare, ai fini del tema trattato, che ciascuna delle quattro nozioni basilari che in sede di analisi possono essere assunte come sottese allo schema (a, b, c, d), è sdoppiata in un termine puro, assolutamente non mescolato al suo opposto (a1, b1, c1, d1), e in un termine non puro, cioè mescolato in qualche modo al suo opposto (a2, b2, c2, d2). Due dei termini non puri $\left(\mathrm{b}_{2}, \mathrm{~d} 2\right)$ sono a loro volta articolati ciascuno in due termini (b2.1, b2.2 e d2.1, d2.2), in una prospettiva che, nel conferire una maggiore definizione a due delle quattro coppie di termini contrapposti e nell'introdurre in due casi la nozione della privazione, si rivela decisiva per la questione dell'esistenza del male. Mentre i termini caratterizzati dalla mescolanza sono tutti - ciascuno a suo modo - esistenti, invece i termini assunti nella loro assolutezza si distinguono in due termini non esistenti (i due termini estremi dello schema, a1 e di) e in due termini esistenti (i due termini intermedi dello schema, b1 e c1), in una visione in cui l'orizzonte dell'esistenza è articolato in otto termini di fondo. Il confronto incrociato tra la distinzione/contrapposizione tra il male assoluto (a1) e il male relativo (a2) e la distinzione/contrapposizione tra il non-essente assoluto (d1) e il non-essente relativo (d2), permette di cogliere, per un verso, il nesso tra il male assoluto (a1) e il non-essente assoluto (d1), e per altro verso, il nesso tra il male relativo (a2) e il non-essente relativo (d2). Quest'ultimo nesso, come vedremo, al di là del suo profilo generico, si specifica come nesso tra il male relativo e il non-essente relativo consistente nella privazione di essere (d2.1), quali termini che Proclo distingue tra loro con riferimento alle corrispettive nozioni di bene e di essente. Inoltre, secondo la mia comprensione, la divisione del bene commisto ad altro (b2) nel bene non mescolato alla privazione (b2.1) e nel bene mescolato alla privazione (b2.2), se qui s'intende la privazione come privazione (parziale) di bene e dunque come male, permette a Proclo, oltre che d'inquadrare il male in termini che a loro modo rimandano alla privazione, anche di stabilire una distinzione, nell'ambito delle realtà derivate dal bene primo, tra ordini in cui il male è totalmente assente e ordini in cui si riscontra il male, distinzione funzionale a confinare quest'ultimo nei soli gradi ipostatici inferiori del reale.

Per quanto concerne il raffronto tra il male in sé e il non-essente assoluto, ritornando al passo sopra citato di $D M S$ 9,1-17, va rilevato che in Proclo il male in sé, se per un verso è concepito come al di là del non-essente assoluto, cioè come ancor più inesistente di quest'ultimo, per altro verso - come ho già mostrato - si rivela perfettamente coincidente con il non-essente assoluto, come risulta dalla parte finale del brano in questione. Questa convergenza tra i due termini, che si risolve nella loro identità, appare chiara se si prendono in esame le loro rispettive configurazioni sul piano concettuale, inquadrandole alla luce della protologia procliana. Per Proclo, posto che non esiste niente che non dipenda dal bene primo e non partecipi in alcun modo della bontà, il male assoluto

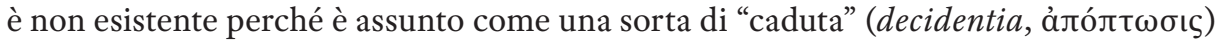

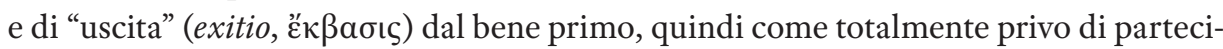


pazione del bene. Ugualmente, posto che non esiste niente che non dipenda dal semplicemente uno e non partecipi in alcun modo dell'unità, il non-essente assoluto (tò $\mu \eta \delta a \mu \tilde{\omega} \varsigma$ őv), cioè il nulla (tò oủ $\varepsilon ́ v$ ), si profila come non esistente perché è concepito come assolutamente separato dal semplicemente uno e dunque, in termini meramente difettivi, come totalmente mancante di unità, la quale s'impone come l'elemento costitutivo primario e imprescindibile di ogni realtà ${ }^{37}$. Secondo Proclo, infatti, il nulla è "ciò che non è neppure uno", secondo la corrispondenza tra tò oủ $\delta \varepsilon v$ (e l'equivalente tò $\mu \eta \delta \varepsilon ́ v$ )

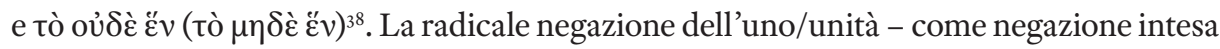
in termini difettivi - è indice di mera non esistenza, dato che per Proclo non può esistere nulla che non possieda in qualche misura un'intrinseca unità riconducibile all'uno in sé, cioè al principio primo, che è solamente uno ${ }^{39}$. Ora, sulla base della dottrina procliana dell'identità protologica del semplicemente uno e del bene primo, come anche dei loro rispettivi effetti, l'unità e la bontà, che sono presenti in tutte le realtà derivate, si può cogliere la coincidenza tra il non-essente assoluto, come assoluta negazione dell'uno/ unità, e il male in sé, come assoluta negazione del bene/bontà. Tanto il primo quanto il secondo, infatti, sono posti, rispettivamente sul versante dell'uno e su quello del bene, all'insegna della totale separazione dal principio primo e dell'assoluta assenza di unitàbontà quale originario carattere riconducibile a quest'ultimo, e da ciò deriva il dato della loro non esistenza. Emerge, così, in modo manifesto l'identità di fondo tra il male in sé e il non-essente assoluto, sottesa al rilievo secondo cui il male in sé è al di là anche del non-essente assoluto, ovverosia è ancor più inesistente di questo. Con tali rilievi, infatti, come abbiamo visto, Proclo non intende porre il male in sé come un più profondo livello di nulla, come un "super-nulla" - oppure, potremmo dire meglio, come un "sub-nulla” ancor più inesistente del nulla stesso, bensì si prefigge di mettere in risalto la non esistenza del male totalmente separato dal bene e di scongiurare il rischio di prospettare una sua inammissibile ipostatizzazione. 5.

${ }^{37}$ Cfr. per es. Procl. Theol.Plat. II 1, 4, 8-21; II 1, 14, 11-12; II 3, 25, 13-22; Procl. in Prm. VI 1081, 10-1082,

38 Cfr. Saffrey, Westerink (2003: t. II, 78-79 nota 7 relativa alla p. 4, part. 78), riguardo a $\mu \eta \delta \varepsilon ́ v$ inteso

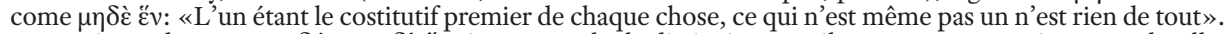

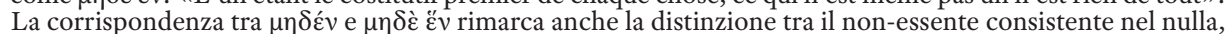
visto come neppure uno, e il non-essente consistente nel principio primo di tutte le cose, visto come l'uno assolutamente semplice, anteriore all'essente; cfr. Procl. in Prm. VI 1081, 8-1082, 7. Su questa distinzione cfr. Guérard (1985).

39 Secondo Proclo, tutto ciò che è altro dall'uno in sé - cioè dal principio primo di tutte le cose - partecipa in qualche modo dell'uno e sussiste come molteplice in quanto è unificato dall'uno, configurandosi, dunque, sia come "non-uno" (oủx čv), in quanto non è solamente uno ed è diverso dall'uno in sé, sia come "uno" ("Év), in quanto è unificato dall'uno e possiede l'unità per partecipazione; cfr. Procl. ET. prop. 1, 2, 1-14 e prop. 2, 2, 15-25. Da ciò emerge l'impossibilità dell'esistenza di qualcosa che sia solamente "non-uno" e non in qualche modo anche "uno". Si profila l'impossibilità dell'esistenza del "nulla" inteso come assoluto "non-uno", come "nessuna cosa" in quanto "neppure uno". Sul piano concettuale, quindi, il nulla si contrappone all'uno; cfr. Procl. Theol.Plat. II 2, 14, 14. 
Questa visione è delineata nei suoi tratti di fondo anche nella Teologia platonica:

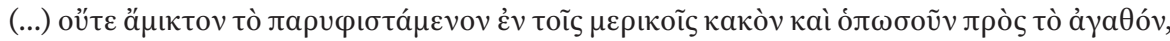

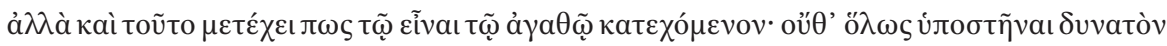

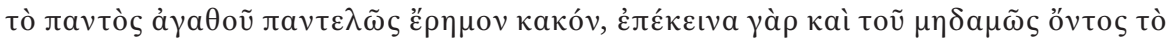

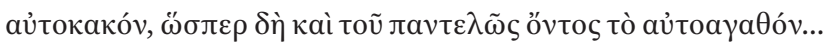

(...) né il male che esiste in modo parassitario nelle realtà particolari è non mescolato - quale che sia il modo - al bene, bensì anche questo partecipa in qualche modo [del bene] per il fatto di essere racchiuso dall'essere del bene; né in generale è possibile che esista il male totalmente separato da ogni bene, perché il male in sé è al di là anche del non-essente assoluto, così come il bene in sé [è al di là] anche del totalmente essente... (Procl. Theol.Plat. I 18, 86, 16-22, trad. mia)

Il male esistente, dunque, è sempre commisto al bene. Questo male, come è noto, è concepito dal Licio non come come anipostatico e neppure come ipostatico, bensì come paripostatico, cioè come esistente per accidente, in altro da sé e in virtù di altro da sé, "presso" qualcosa d'ipostatico, secondo una modalità di esistenza collaterale e parassitaria rispetto all'esistenza ipostatica di ciò in cui il male si manifesta ${ }^{40}$. Nel pensiero procliano,

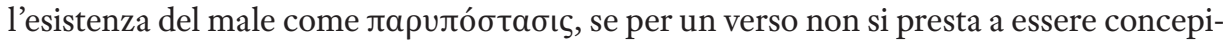
ta come una contro-esistenza, intendendo con ciò un'esistenza ontologicamente "forte" e protologicamente opposta al bene primo, per altro verso non va neppure concepita come una pseudo-esistenza, intendendo con ciò una mera parvenza illusoria di esistenza, priva di una fattuale incidenza sulla trama generale dell'esistente. In quest'ultimo caso, infatti, sul piano metafisico, il male commisto al bene sarebbe pensato anch'esso come non esistente e finirebbe in ultima istanza per coincidere con il male in sé; così, insomma, si annullerebbe la distinzione tra il male relativo-paripostatico e il male assoluto-anipostatico, con la riduzione del primo al secondo, e si cadrebbe in quella stessa tesi della non

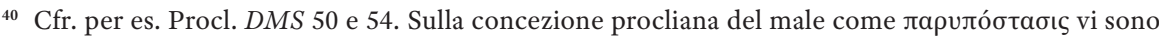
numerosi studi, che offrono varie prospettive di lettura; tra questi, cfr. Rosán (1949: 180-181); Beierwaltes (1973: 157-158); Isaac (2003b: 12-17); Lloyd (1987); de Libera (1993: 100-101); Abbate (1998); Opsomer, Steel (2003: 24-28; 2017: 248-251); Paparella (2008: 54 ss.); Skliris (2008: part. 152-155); Kavvadas (2009: part. 81-82; 2015); Chlup (2009: 27-28; 2012: 208-211); Charrue (2010: 129-131); Nikulin (2016: 143-145); Moraru (2019). Per Proclo, il male è mancante di una propria ipostasi ed esiste solo in rapporto all'ipostasi di ciò in cui esso attecchisce, in forza dello sfruttamento della potenza del bene che è proprio delle ipostasi. Assunto in termini di ларило́бтабıৎ, il male non ha alcuna causa propria e principale, bensì si configura come il prodotto deviato e deficitario di una causalità meramente accidentale, come un effetto collaterale di una causa rivolta per se stessa a un bene che, come fine naturale, non viene però raggiunto per deficienza o debolezza (cfr. Procl. DMS 40 e 50). Su quest'ultimo aspetto cfr. Bonazzi (2015: 141); Opsomer, Steel (2003: 26); Paparella (2004: 81-82); Linguiti (2012: 251). Così concepito, il male, a fronte dell'inesistenza di qualcosa che sia il "contrario" (contrarium, tò Évavtíov) del bene primo (cfr. Procl. DMS 37, 31-32), è posto come subcontrario (subcontrarium, úrcvavtíov) del bene (cfr. Procl. DMS 54, 29), perché in ogni caso è opposto soltanto a un bene determinato e particolare, di cui è privazione, e non al bene primo e alla totalità dei beni. Secondo Proclo, inoltre, il male, così concepito, ha un'esistenza che potremmo definire periferica, dato che esso è assente nei più elevati ordini ipostatici, cioè negli dèi, come anche negli angeli, nei demoni, negli eroi e, in ultima istanza, nelle anime divinizzate (cfr. Procl. $D M S$ 11-21), e si manifesta soltanto nelle anime particolari e nei corpi particolari (cfr. Procl. DMS 4 e 5). Come tale, il male è connesso con il difetto di potenza che è riscontrabile solo negli ordini più bassi, in cui la molteplicità è causa di allontanamento dall'unità. 
esistenza tout court del male, dalla quale Proclo intende prendere le distanze. L'esistenza del male, se nel pensiero dello Scolarca si presenta come metafisicamente "debole", rappresenta pur sempre una data modalità di esistenza, che egli considera non soltanto come pienamente compatibile con le istanze e le implicazioni della sua protologia monistica agatologica ${ }^{41}$, ma anche come imprescindibile per la configurazione di quest'ultima. Nella visione procliana, infatti, il male è concepito come un aspetto necessario che sul piano cosmologico concorre a configurare la perfezione del mondo fisico, e in una più ampia ottica metafisica concorre a determinare il rango e la funzione causale degli ordini divini, come anche l'articolazione gradazionale della realtà ${ }^{42}$.

\section{Il male alla luce della dottrina procliana della multivocità del non-essente}

Nel pensiero procliano, la modalità di esistenza riconosciuta al male si presta a essere inquadrata in termini meontologici. Abbiamo visto come nel $₫ 9$ del De malorum subsistentia la distinzione tra il male in sé e il male mescolato al bene sia elaborata con rimando alla distinzione tra il non-essente assoluto e il non-essente relativo, la quale costituisce un assunto portante della dottrina procliana della multivocità del non-essente (tò $\mu$ ì őv $)^{43}$. In Proclo il non-essente assoluto, ciò che non è in alcun modo ( assunto come nozione rigorosamente univoca, è appunto cifra della totale negazione dell' "essere” inteso nel suo più esteso senso esistenziale, e indica esclusivamente la totale assenza di qualsivoglia ordine e modalità di esistenza, cioè il nulla, come termine che, nella sua assolutezza, è impossibile e immaginario ${ }^{44}$. Di contro, il non-essente relativo, posto in contrapposizione al non-essente assoluto, manifesta un'intrinseca molteplicità di significati, cioè si articola in una pluralità di tipi di non-essente che, nella concezione

${ }^{41}$ La dottrina procliana del male, che in linea di principio comporta una concezione dei mali del corpo e di quelli dell'anima alla luce dell'unico modello teorico della modalità paripostatica di esistenza, se da un lato è presentata da Proclo come risolutiva della questione del male sia sul piano cosmologico-metafisico sia su quello etico, dall'altro lato, in sede di analisi critica si rivela non priva di tensioni interne e di possibili risvolti aporetici. Per alcune stimolanti osservazioni su aspetti aporetici della dottrina procliana del male cfr. per es. Paparella (2008: 59-61, 65-67, 95-96) e Kavvadas (2015).

42 Su questi rilievi cfr. Procl. DMS 5, 7-34; 7; Procl. Dec. dub. V 28-29; Procl. in Ti. I 372, 20-373, 3.

43 Sulla multivocità del non-essente in Proclo cfr. per es. Procl. in Prm. V 999, 16-1000, 25; VI 1039, 24-25; VI 1073, 2-4; VII 503, 21-23; Procl. Theol.Plat. II 5, 38, 26-39, 5; Procl. DMS 8, 26-38; 9, 1-5; Procl. in Ti. I 233 , 1-4. Tra gli studi, cfr. Narbonne (1992: 123-124); Pinchard (2006; 2007); Phillips (2007: 80-82); Napoli (2008: 313-330); de Garay (2013); Luna, Segonds (2014: part. 166-172, dalla nota 4 relativa alla p. 36 alla nota 8 relativa alla p. 38); Del Forno (2015: part. 246-247).

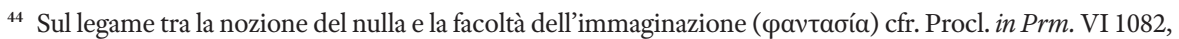
7-12; VI 1105, 25-1106, 1. 
gradazionale del reale propria del pensiero procliano, rappresentano diverse modalità di esistenza, corrispondenti a vari ordini o aspetti del reale ${ }^{45}$.

In un passo del Commentario sul "Parmenide" Proclo delinea un elenco tassonomico di diversi tipi di non-essente. Egli presenta in termini di non-essente, secondo diverse configurazioni, oltre al non-essente assoluto (tò $\mu \eta \delta \alpha \mu \tilde{\eta} \mu \eta \delta \alpha \mu \tilde{\omega} \varsigma$ ő $v$, che è espressione concettualmente equivalente a tò $\mu \eta \delta \alpha \mu \tilde{\omega} \varsigma$ ǒ $v$ ) e alla privazione (puntualmente distinta dal non-essente assoluto), i vari ordini gradazionali del reale, ciascuno visto nei tratti peculiari della sua strutturazione: la materia, le forme legate a questa, le cose sensibili, le anime, il genere intelligibile del diverso, il principio primo di tutte le $\operatorname{cose}^{46}$. A differenza del non-essente assoluto, gli altri tipi di non-essente prospettati in questo scenario, diversi tra loro, sono tutti pensati, ciascuno a suo modo, sotto il segno dell'esistenza e si prestano quindi a essere inquadrati come diverse articolazioni di ciò che potremmo delineare, in una prospettiva generale, come il non-essente assunto in senso relativo. La materia, le forme unite alla materia, le cose sensibili e le anime, configurate in diversi modi come non-essenti, sono tutte inferiori, secondo diversi gradi, ai "realmente essenti", cioè alle realtà intelligibili, immutabili ed eterne. Su un piano superiore, il non-essente si riscontra anche nella stessa realtà intelligibile ed è individuato nella "prima diversità degli essenti", cioè nel genere del diverso, il quale, con riferimento a un assunto platonico del Sofista, non è essente in misura minore dello stesso essente ${ }^{47}$. Al di sopra di tutti questi ordini meontologici, è posto come non-essente anche il principio primo di tutte le cose, in quanto è superiore all'essente ${ }^{48}$, in termini di assoluta trascendenza, che è anche anteriorità causale. Tra i vari tipi di non-essente relativo, a ridosso dei più bassi ordini del reale,

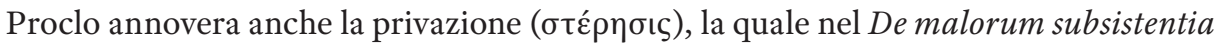
si rivela un termine di riferimento basilare della concezione procliana del male. Va anche rilevato che in Proclo, al di là di quanto si legge nel passo in questione di in Prm. V 999, si configurano come non-essenti anche gli altri intelligibili, ciascuno dei quali "non è" gli altri, cioè è diverso da tutti gli altri per la sua partecipazione del genere del diverso (la sopra menzionata "prima diversità degli essenti"), che svolge la funzione di principio di separazione e di non commistione ${ }^{49}$. Il Diadoco, inoltre, concepisce in termini meonto-

45 Secondo la mia comprensione, Proclo, a fronte della delineazione di una pluralità di tipi di non-essente relativo, talvolta profila anche una contrapposizione di fondo tra il non-essente assoluto, visto nella sua univocità, e il non-essente relativo assunto in una prospettiva generale, con riferimento al suo carattere di esistenza, a prescindere dalla varietà tipologica dei termini meontologici in cui si articola. Cfr. per es. Procl. in Prm. V 1035, 11-12.

${ }^{46}$ Cfr. Procl. in Prm. V 999, 16-31 e più in generale 999, 9-1000, 25.

${ }^{47}$ Cfr. Procl. in Prm. V 999, 27-29. Il riferimento è a Pl. Sph. 258a11-b2.

${ }_{48}$ Per Proclo, il principio primo, visto nella sua trascendenza rispetto a tutte le cose e nella sua ineffabilità, non è neppure non-essente ed è al di sopra anche del non-essente; cfr. Procl. in R. I 282, 21-25. Cfr. anche Procl. Theol.Plat. II 5, 37, 25-38, 1, in cui l'uno è presentato come il principio degli essenti e dei non-essenti. Va anche ricordato che, per il Licio, al principio primo non si addicono in senso proprio neppure le negazioni dal valore trascendente e causale, le quali vanno anch'esse negate. Sulla negazione della negazione in Proclo cfr. Jugrin (2017: part. 184-189).

49 Cfr. Procl. in Prm. VII 1178, 25-27; Procl. in Ti. II 158, 20-159, 3. Cfr. anche Procl. in Prm. VII 503, 21-23, in cui il filosofo, nel delineare una classificazione selettiva di quattro significati di non-essente, vi 
logici anche le enadi, che nel passo di in Prm. V 999 non sono menzionate; queste, nel loro complesso, sono inquadrabili come un'articolazione del non-essente al di là dell'essente, in quanto sono concepite come sovraessenziali, ancorché secondo una modalità differente e inferiore rispetto a quella del principio primo di tutte le cose, dato che, mentre questo è totalmente impartecipabile, invece le enadi sono partecipate dagli essenti ${ }^{50}$.

Proclo, così, con riferimento alla nozione generale del non-essente relativo, delinea diversi tipi di non-essenti, tutti accomunati dall'esistere in qualche modo e, dunque, dal distinguersi dal non-essente assoluto, puramente non esistente, quali diversi ordini di realtà che potremmo indicare come i non-essenti subessenziali (la materia, le realtà materiali e sensibili, le anime), i non-essenti essenziali (il genere intelligibile del diverso, ma anche, sulla base della partecipazione di questo, i generi e le forme intelligibili) e i non-essenti sovraessenziali (il principio primo e, in diverso modo, le enadi). Nel caso del non-essente assunto in senso relativo, infatti, la negazione dell'essente si presenta secondo tre diverse linee di orientamento: come cifra d'inferiorità rispetto all'essere essenziale; come cifra differenziale interna all'articolato ordinamento dell'essere essenziale, in una prospettiva in cui il non-essente è posto come congiunto e coordinato all'essente $^{51}$; come cifra di superiorità rispetto all'essere essenziale ${ }^{52}$. La privazione, che in questo quadro d'insieme è posta sul versante dell'inferiorità rispetto all'essente essenziale, non rappresenta un dato ordine ipostatico, ma un aspetto difettivo riscontrabile in taluni ordini ipostatici.

Con riferimento a questo scenario speculativo, in Proclo, mentre il male in sé è accostato al non-essente assoluto per il fatto di essere posto come non esistente, invece il male mescolato al bene è pensato in corrispondenza del non-essente relativo con riferimento alla comune cifra di esistenza. Riguardo a quest'ultima corrispondenza, mi sembra che si possano distinguere due diversi aspetti di essa. Per un verso, in una prospettiva generale, la corrispondenza tra il male mescolato al bene e il non-essente relativo si basa sul fatto che entrambi i termini sono posti come esistenti, dunque è posta con riferimento al non-essente relativo visto in modo generico come esistente, in contrapposizione al non-

include il non-essente consistente nei generi intelligibili della quiete e del movimento, presentabili entrambi in termini di non-essente «per via della natura del diverso», cioè per la loro partecipazione del genere intelligibile del diverso. In questo caso, dunque, il non-essente essenziale è indicato nei generi della quiete e del movimento, i quali, come gli altri generi e forme intelligibili, si configurano come non-essenti in virtù della loro partecipazione del genere del diverso. La quiete, infatti, "non è" il movimento, così come "non è" tutti gli altri generi e forme, e il movimento "non è" la quiete, così come "non è" tutti gli altri generi e forme, nel senso che ciascuno di questi due generi, nella propria determinatezza, è diverso da tutti gli altri. I due generi suddetti, dunque, sono presentati come casi esemplari di una configurazione meontologica che accomuna gli intelligibili. Proclo, infatti, poco dopo, nel parlare di questo tipo di non-essente, fa riferimento alla quiete, al moto e, in generale, al non-essente che è nell'ambito degli intelligibili, ovverosia al non-essente che ciascun intelligibile è in virtù del fatto che "non è" tutti gli altri intelligibili, cioè "è diverso" da questi.

${ }^{50}$ Per questi rilievi cfr. Procl. in Prm. VI 1073, 9-11; Procl. ET. prop. 116, 102, 13-27.

${ }^{51}$ Cfr. Procl. in Prm. VI 1072, 24-1073, 1, da cui si può evincere che il filosofo, con riferimento al passo di Pl. Sph. 258a11-b2, stabilisce pur sempre la superiorità dell'essente rispetto al non-essente che non è meno essente dell'essente, nell'ambito dell'articolazione degli ordini ontico-noetici.

52 Cfr. Procl. in Prm. VI 1073, 2-4; Procl. Theol.Plat. II 5, 38, 26-39. 
essente assoluto (cfr. Procl. DMS 9, 1-17). Per altro verso, guardando alla specificità delle articolazioni tipologiche in cui si dà il non-essente relativo, la suddetta corrispondenza si determina con particolare riferimento al tipo di non-essente relativo consistente nella

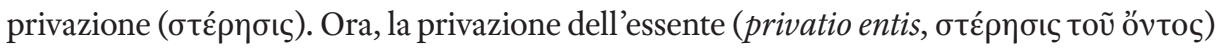
è una delle due determinazioni tipologiche in cui nel $\$ 8$ del De malorum subsistentia è articolato il non-essente commisto all'essente, ovverosia il non-essente relativo, in una classificazione dicotomica di quest'ultimo termine che, nella sua impostazione selettiva, è calibrata in funzione della trattazione della specifica questione che lo Scolarca affronta in questa parte dell'opusculum (cfr. Procl. DMS 8, 31-33).

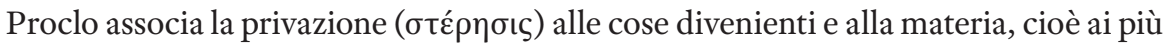
bassi ordini della realtà ${ }^{53}$. La privazione, in senso proprio, non s'identifica con le cose divenienti e con la materia, alle quali è legata ${ }^{54}$, ma costituisce un carattere che contraddistingue questi ordini ipostatici. Come tale, essa si configura come una modalità di esistenza consistente non in uno specifico ordine ipostatico, bensì in una costitutiva condizione difettiva riscontrabile nel modo di essere di alcuni ordini ipostatici. La privazione, infatti, consiste nella mancanza difettiva di un determinato stato o proprietà, riscontrabile in un termine ipostatico che funge da sostrato tetico d'inerenza e che è passibile di una siffatta condizione. Sulla scia di un assunto aristotelico, Proclo sostiene che la privazione "per sé"

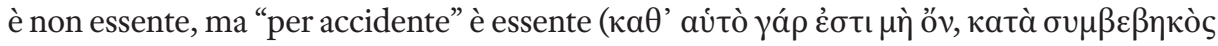
$\delta \varepsilon$ ô $\left.^{5}\right)^{55}$. La privazione, infatti, mentre di per sé è non essente perché è indice dell'assenza di una determinata proprietà in una realtà soggiacente, invece per accidente si configura come essente perché indica il non sussistere di una data proprietà in qualcosa di essente cui essa in ogni caso si riferisce, e in tal senso contribuisce anch'essa a caratterizzare lo stato di qualcosa di essente. Come cifra di mancanza di possesso di qualcosa, registrabile in una data cosa, la privazione presuppone l'esistenza di ciò in cui essa si riscontra, cioè l'essere della cosa senza cui la privazione non potrebbe neppure darsi come tale. Così, la privazione è anch'essa essente, ancorché in modo accidentale, lasciandosi pensare sotto il segno della negazione relativa dell'essere e presentandosi come un tipo di non-essente relativo, esistente ma non ipostatico.

Ora, secondo Proclo, il male, posto come esistente, è privazione parziale di bene, cioè privazione di qualche bene particolare riscontrabile in qualcosa, e non privazione del bene primo e di ogni bene in assoluto. Come tale, il male, esistendo soltanto in altro e in virtù di altro, al pari del tipo di non-essente relativo consistente nella privazione,

${ }_{53}$ Cfr. Procl. Theol.Plat. II 5, 39, 2-5, in cui il Diadoco parla del non-essente che è privazione dell'essente

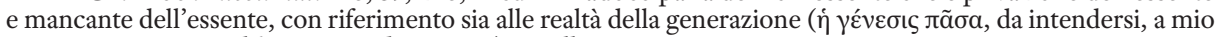
avviso, come ogni realtà generata-diveniente), sia alla materia.

${ }_{54}$ Proclo, in in Prm. V 999 16-31, presenta la privazione come un tipo di non-essente relativo distinto sia dalla materia sia dalle cose divenienti, introducendola per prima in un elenco di tipi di non-essente relativo che, con riferimento a una divisione gerarchizzata di tipi di esistenza, è ordinato dal basso verso l'alto.

55 Cfr. Procl. in Prm. V 999, 18-19. Questa tesi è formulata sulla scorta di una rielaborazione di assunti aristotelici; cfr. Arist. Ph. I 7, 190b24-30; I 8, 191b15-16; I 9, 192a3-6 Carteron; Metaph. IV 2, 1004a12-16; V 22, 1022b22-1023a7 Ross. 
non possiede una propria sussistenza autonoma, una "perseità", e si configura come un non-essente non ipostatico, esistente non come un dato ordine di realtà, bensì come un aspetto riscontrabile in certi ordini di realtà. Va però precisato che, al di là di tale convergenza, per Proclo il male non può essere semplicemente assimilato alla privazione assunta in quanto tale, dato che esso si configura come privazione di bene e non come privazione di essere ${ }^{56}$. Nella visione procliana, stabilito che sul piano concettuale la privazione è il contrario dell'essere e che il male è il contrario del bene, la privazione e il male sono posti come diversi perché diversi sono i loro rispettivi termini opposti, l'essere e il bene. L'anteriorità del bene rispetto all'essere, la quale esclude l'identità tra i due termini e la loro conversione reciproca, implica che la presenza della privazione, che è relativa all'essere, non comporta di per se stessa la concomitante presenza del male, che invece è relativo al bene. Inoltre, nel caso del male, a differenza delle privazioni ontiche, se la privazione diviene totale, essa conduce alla completa dissoluzione dell'ipostasi cui è riferita, e nel caso specifico comporta la scomparsa anche del male ${ }^{57}$. Alla luce dell'anteriorità del bene rispetto all'essere, il male, che è privazione di bene, non può essere posto come esistente se non come privazione parziale di bene, poiché la totale assenza di bene implica totale assenza di essere, ovverosia la mera non esistenza ${ }^{58}$. Inoltre, le privazioni ontiche, di per se stesse, sono mere assenze di proprietà, e come tali in senso proprio non traggono esistenza dalle cose cui si riferiscono e non si oppongono alle cose di cui sono soppressioni e semplici negazioni, non manifestando in tal modo alcuna potenza e attività; il male, invece, come la privazione del bene, si oppone al bene di cui è privazione e trae potenza dal bene cui è mescolato, per rafforzare la propria inconsistenza e per combatterlo. Così, a differenza delle privazioni ontiche, il male manifesta una natura parassitaria, cioè esiste soltanto in presenza del bene, configurandosi come una privazione parziale di questo. Infatti, mentre le altre privazioni si danno quando in un soggetto è totalmente assente lo stato di cui ciascuna di esse è privazione, invece la privazione di bene non può esistere in assenza totale di quest'ultimo, quale assenza che comporta la non esistenza del soggetto stesso che funge da sostrato ${ }^{59}$.

In Proclo, dunque, l'accostamento del male al peculiare non-essente relativo consistente nella privazione, con la delineazione della strutturale differenza che intercorre tra i due termini, appare funzionale a non conferire alcuna perseità ipostatica al male, che pure è posto come esistente. La distinzione tra la privazione, assunta come privazione dell'essente, e il male, posto come privazione del bene, permette di assegnare all'esisten-

${ }^{56}$ Sulla tesi procliana della differenza tra la privazione ontica e il male cfr. Procl. DMS 38, 1-45; 7, 35-69; 52, 1-41. Tra gli studi, cfr. Opsomer, Steel (2003: 29-31); Steel (1998: 92-97; 2017: 251-253); Fiorentino (1998: 78-79); Phillips (2007: 57-90).

57 Questo assunto è esemplificato con il caso della malattia, la quale esiste nel corpo se essa è un disordine parziale, dato che la privazione completa dell'ordine conduce alla morte del corpo e, con questa, alla cessazione della malattia, cioè distrugge il soggetto insieme al male che è in esso; cfr. Procl. DMS 38, 14-24. Sull'esempio della malattia cfr. anche Procl. DMS 7, 63-68.

58 Sul fatto che il male a suo modo esistente non è privazione totale di bene, cfr. Procl. DMS 54, 25-29.

59 Per questi rilievi cfr. Procl. DMS 7, 38-57; 38, 10-45; 52, 3-14. 
za del male caratteri specifici che s'inquadrano in un orizzonte non ontologico, bensì agatologico, come anche di non ricondurre ogni privazione al male.

\section{Per concludere}

Nella dottrina procliana del male riveste una basilare importanza la recisa negazione dell'esistenza del male in sé. Inquadrato nel suo rapporto di corrispondenza inversa con il bene primo, il male in sé si configura come "al di là anche del non-essente assoluto", e ciò significa che il male in sé, come il nulla, non esiste in alcun modo. Questo assunto emerge chiaramente anche da un'altra formulazione procliana della corrispondenza inversa del male in sé con il bene primo: come il bene primo è al di là di tutte le cose - o, si potrebbe anche intendere, al di là di tutti i beni -, così il male in sé è privo della partecipazione di tutti i beni, ovverosia è separato da ogni bene, ed è difetto e privazione di tutti questi (ut enim le prime bonum ultra omnia, sic malum ipsum omnium expers bonorum, dico autem qua malum, et defectus illorum et privatio $)^{60}$. Anche questo rilievo mostra la non esistenza del male in sé, in una visione in cui non può esistere niente che non partecipi in qualche modo del bene. Esistente, invece, è il male assunto come privazione parziale del bene, cioè come male mescolato al bene, ed è un male che, nelle realtà in cui si trova, non può mai sovrastare il bene cui è commisto. Così configurato, il male, presente solo nei più bassi ordini del reale, è concepito come integrato nella strutturazione agatologica dell' intera realtà e come conciliabile con il dominio incontrastato della

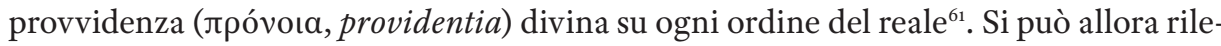
vare che in Proclo il male si lascia pensare in una visione filosofica che potremmo classificare come ottimistica ${ }^{62}$. La tesi della sola esistenza del male commisto al bene, intesa come esistenza di una fenomenologia di mali paripostatici, comporta una relativizzazione del male, speculativamente disinnescato nei suoi aspetti più tetri. Si delinea, così, uno scenario in cui Proclo, che intende mantenere la distinzione tra il bene in quanto tale e il male, può anche giungere ad affermare, in una visione filosofica generale, che il male non soltanto esiste in virtù del bene, ma è anche un bene per il fatto di esistere ${ }^{63}$, è un bene, potremmo dire, nel suo stesso esistere come male in virtù del bene, in quanto l'esistenza come tale è intrinsecamente legata al bene. Con questo suo statuto, il male

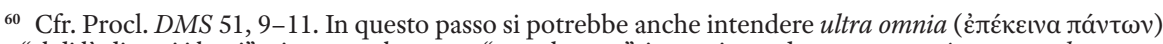
come "al di là di tutti i beni", piuttosto che come "tutte le cose", in corrispondenza con omnium expers bonorum

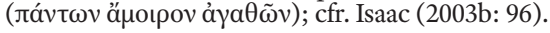

${ }^{61}$ Sulla conciliabilità tra il male concepito come mescolato al bene e la provvidenza universale cfr. per es. Procl. DMS 58-61.

${ }^{62}$ Cfr. Lanzi (2000: 282-283); Brogi (2006: 50-51); García Valverde (2017: 149).

${ }_{63}$ Cfr. Procl. DMS 5, 36-37. Questo rilievo, formulato in una specifica prospettiva cosmologica (cfr. Procl. DMS 5, 34-36) nella parte iniziale dell'opusculum, nell'ambito degli argomenti esposti a favore della tesi generale dell'esistenza del male, nella sua somma paradossalità appare di fatto conservato e spiegato da Proclo nella sua visione generale del male (cfr. DMS 5, 37-38). Cfr. per es. anche DMS 58-61. 
commisto al bene rientra anche in una protologia volta a negare l'esistenza di un principio dei mali contrapposto al bene primo. Nella filosofia procliana, tra il bene e il male non vi è alcuna opposizione dualistica né alcun conflitto protologico. Per il Licio, infatti, il principio primo non può che essere uno e unico, e questo principio è il bene primo, il quale, proprio in quanto tale, è la causa prima di tutti i beni e non può essere posto anche come la causa del male, né può comportare che quest'ultimo sia prodotto da una qualche causa propria, dato che tutte le cause sono subordinate al bene primo, nella loro potenza causale, dipendono da questo. In tale visione monistica, l'uno-bene primo, nella sua assolutezza, non ha alcun reale termine contrario: il suo contrario, ciò che non è neppure uno, o il male puro, non è nient'altro che il nulla, quale termine che per definizione è totalmente inesistente e impossibile. Nel porre il male come patipostatico, dunque, Proclo evita sia di negare l'esistenza del male in qualsivoglia modalità, sia di attribuire al male una natura propria e a se stante. Egli, insomma, mostra di volersi tenere lontano sia da una mera nientificazione del male, che condurrebbe all'abolizione della differenza stessa tra il bene e il male nei termini di una piena coincidenza del secondo con il primo, sia da qualsivoglia forma d'ipostatizzazione del male, che comporterebbe la negazione del monismo agatologico e delle sue implicazioni.

In questo scenario, con riferimento alla questione del male, in Proclo lo spettro dei diversi significati del non-essente si arricchisce di due termini: sul versante esistenziale vi si annovera il male paripostatico, descritto come un tipo di privazione non riducibile alla privazione ontica, e sul versante della radicale negazione dell'esistenza vi si registra il male assoluto, il quale, seppure in una certa ottica sia distinguibile nella nozione dal non-essente in senso assoluto, coincide in ultima istanza con questo. 


\section{BIBLIOGRAFIA}

Abbate, M., 1998, “Parypóstasis: il concetto di male nella quarta dissertazione del Commento alla Repubblica di Proclo", Rivista di Storia della Filosofia 53, pp. 109-115.

BechtLe, G., 1999, “Das Böse im Platonismus: Überlegungen zur Position Jamblichs”, Bochumer Philosophisches Jahrbuch für Antike und Mittelalter 4, pp. 63-82.

Beierwaltes, W., 1973, “Die Entfaltung der Einheit. Zur Differenz plotinischen und proklischen Denkens”, Thêta-Pi 2/2, pp. 126-161 [anche in: W. Beierwaltes, Denken des Einen: Studien zur neuplatonischen Philosophie und ihrer Wirkungsgeschicht, Frankfurt am Main 1985].

Beierwaltes, W., 2004, “Proklos' Begriff des Guten aus der Perspektive seiner Platon-Deutung”, in: Kijewska (2004), pp. 97-120 [anche in : W. Beierwaltes, Procliana. Spätantikes Denken und seine Spuren, Frankfurt am Main 2007, pp. 85-108].

Beierwaltes, W., 2014³ , Proklos. Grundzüge seiner Metaphysik, Frankfurt am Main [rist. della 2a ed., 1979].

BoEse, H. (ed.), 1960, Procli Diadochi Tria opuscula (De providentia, libertate, malo), Latine Guilelmo de Moerbeka vertente et Graece ex Isaacii Sebastocratoris aliorumque scriptis collecta, Berlin.

BonazzI, M., 2015, Il platonismo, Torino.

Boss, G., SeEL, G. (eds.), 1987, Proclus et son influence. Actes du Colloque de Neuchâtel, Juin 1985, Zürich.

Bossi, B., Robinson, TH. M. (eds.), 2013, Plato's Sophist Revisited, Berlin-Boston.

Brogi, S., 2006, I filosofi e il male. Storia della teodicea da Platone a Auschwitz, Milano.

Burnet, J. (ed.), 1900-1907, Plato, Opera, vol. I-V, Oxford.

CARDullo, R. L., 2017, "Evil as alogon in the post-Plotinian Neoplatonism. Again on the Uniqueness of the Principle, between Athens and Alexandria”, in: Cardullo, Coniglione (2017), pp. 47-63.

Cardullo, R. L., Coniglione, F. (eds.), 2017, Reason and No-reason from Ancient Philosophy to Neurosciences. Old Parameters, New Perspectives, Sankt Augustin.

Carteron, H. (ed.), 1926-1932, Aristote, Physique, vol. I-II, Paris.

Charrue, J.-M., 2010, De l'être et du monde. Ammonius, Plotinus, Proclus, Paris.

Chiaradonna, R. (cur.), 2012, Filosofia tardoantica, Roma.

Chlup, R., 2009, “Proclus' Theory of Evil: An Ethical Perspective”, The International Journal of Platonic Tradition 3, pp. 26-57.

Chlup, R., 2012, Proclus. An Introduction, Cambridge-New York.

D’Hoine, P., 2011, “Les arguments de Proclus contre l'existence d'Idées des maux”, Études Platoniciennes 8, pp. 75-103.

D’Hoine, P., Martuj, M. (eds.), 2017, All from One. A Guide to Proclus, Oxford-New York.

DE ANdia, Y. (éd.), 1997, Denys l’Aréopagite et sa posterité en Orient et en Occident. Actes du Colloque International, Paris, 21-24 septembre 1994, Paris.

DE Garay, J., 2013, “Difference and Negation: Plato's Sophist in Proclus”, in: Bossi, Robinson (2013), pp. 225-245.

DE LibERA, A., 1993, “Le mal dans la philosophie médiévale”, Studia Philosophica 52, pp. 81-103.

DeL Forno, D., 2015, La dialettica in Proclo. Il quinto libro dell'In Parmenidem tradotto e commentato, Sankt Augustin.

DieHL, E. (ed.), 1903-1906, Proclus Diadochus, In Platonis Timaeum commentaria, vol. I-III, Leipzig.

DoDDs, E. R. (ed.), 1963², Proclus, The Elements of Theology, Oxford-New York. 
ErLer, M. (tr.), 1978, Proklos Diadochos, Über die Existenz des Bösen, Meisenheim am Glan.

Festugière, A. J. (tr.), 2006, Proclus, Commentaire sur le Timée, t. II - Libre II, Paris [1967¹].

Finamore, J. F., KLITENic WeAR, S. (eds.), 2017, Defining Platonism. Essays in Honor of the 75th Birthday of John M. Dillon, Steubenville.

Fiorentino, F., 1998, “Il male nel neoplatonismo”, Idee 37-38, pp. 61-84.

Fuhrer, Th., Erler, M. (Hg.), 1999, Zur Rezeption der hellenistischen Philosophie in der Spätantike. Akten der 1. Tagung der Karl-und-Gertrud-Abel-Stiftung vom 22.-25. September 1997 in Trier, Stuttgart.

García VAlverde, J. M. (tr.), 2017, Proclo, Elementos de teología. Sobre la providencia, el destino y el mal, Madrid.

GuÉRARD, CH., 1985, “Le danger du néant et la négation selon Proclus”, Revue Philosophique de Louvain 83, pp. 331-354.

HenRY, P., Schwyzer H.-R. (eds.), 1964-1983, Plotinus, Opera, vol. I-III, Oxford-New York.

IsAAC, D. (ed.), 2003a² , Proclus, Trois études sur la providence, t. I: Dix problèmes concernant la Providence, Paris $\left[1977^{1}\right]$.

IsAAC, D. (ed.), 2003b² , Proclus, Trois études sur la providence, t. III: De l'existence du mal, Paris [1982 $\left.{ }^{1}\right]$.

Jugrin, D., 2017, Negation and Knowledge of God: Neoplatonism and Christianity, Beau Bassin.

Kavvadas, N. CH., 2009, Die Natur des Schlechten bei Proklos. Eine Platoninterpretation und ihre Rezeption durch Dionysios Areopagites, Berlin-New York.

Kavvadas, N. CH., 2015, "Beyond Parhypostasis? A Negation of the Existence of Extra-Moral Evil in Proclus and its Contexts", Dionysius n.s. 33, pp. 179-194.

KuJEwska, A. (ed.), 2004, Being or Good? Metamorphoses of Neoplatonism, Lublin.

KrolL, W. (ed.), 1899-1901, Proclus Diadochus, In Platonis Rem publicam commentarii, vol. I-II, Leipzig.

LANzI, S., 2000, Theos Anaitios, Storia della teodicea da Omero ad Agostino, Roma.

Laurent, J., Romano, C. (eds.), 2006, Le Néant. Contribution à l'histoire du non-être dans la philosophie occidentale, Paris.

Linguiti, A., 2012, “Etica”, in: Chiaradonna (2012), pp. 233-252.

Lloyd, A. C., 1987, “Parhypostasis in Proclus”, in: Boss, Seel (1987), pp. 145-157.

Luna, C., Segonds A.-PH. (eds.), 2014, Proclus, Commentaire sur le Parménide de Platon, t. V, Paris.

Maurette, P., 2004, “El mal y lo ilimitado en la filosofía de Proclo”, Nova Tellus 22, pp. 141-165.

Montoneri, L. (tr.), 1986, Proclo, La provvidenza e la libertà dell'uomo, Roma-Bari.

Moraru, C.-F., 2019, “The Concept of Parhypostasis in Proclus' De malorum subsistentia. A Meontological Perspective”, Revue Roumaine de Philosophie 63/1, pp. 71-85.

Moutsopoulos, E., 2006, “'Елع́кєıva. L’idée de la transcendance dans la philosophie de Proclus”, Diotima 34, pp. 144-152.

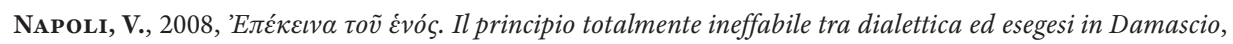
Catania-Palermo.

NArbonne, J.-M., 1992, "Le non-être chez Plotin et dans la tradition grecque”, Revue de Philosophie Ancienne 10, pp. 115-133.

Nikulın, D., 2016, “Proclus on Evil”, Proceedings of the Boston Area Colloquium in Ancient Philosophy 31.1, pp. 119-146 e 156-158 [con qualche modifica, anche in: D. Nikulin, Neoplatonism in Late Antiquity, OxfordNew York 2019, pp. 177-203]. 
O’Brien, D., 2017, "Plotinus on Evil. Proclus and the Author of The Divine Names", in: Finamore, Klitenic Wear (2017), pp. 130-161.

Opsomer, J., Steel C., 1999, “Evil without a Cause: Proclus' Doctrine of the Origin of Evil, and its Antecedents in Hellenistic Philosophy”, in: Fuhrer, Erler (1999), pp. 229-260.

Opsomer, J., STEel, C. (tr.), 2003, Proclus, On the Existence of Evils, Ithaca (NY).

Paparella, F. D. (tr.), 2004, Proclo, Tria opuscula. Provvidenza libertà male. Dieci questioni sulla provvidenza. Lettera all'inventore Teodoro sulla provvidenza, il fato e ciò che è sotto il potere dell'uomo. Sull'esistenza del male, Milano.

Paparella, F. D., 2008, "La teodicea di Proclo e il suo rapporto con la teodicea antica e tardo-antica", Mediaevalia. Textos e estudos 27, pp. 47-97.

Phillips, J., 2007, Order from Disorder. Proclus' Doctrine of Evil and its Roots in Ancient Platonism, Leiden-Boston.

Pinchard, A., 2006, “Proclus”, in: Laurent, Romano (2006), pp. 141-162.

Pinchard, A., 2007, “Négation et privation dans l'In Parmenidem de Proclus", Cahiers de Philosophie de l'Université de Caen» 43, pp. 85-93.

Rosán, L. J., 1949, The Philosophy of Proclus. The Final Phase of Ancient Thought, New York.

Ross, W. D. (ed.), 1924, Aristotle, Metaphysics, vol. I-II, Oxford.

SAffrey, H. D., Westerink, L. G. (eds.), 2003², Proclus, Théologie platonicienne, vol. I-VI., Paris [1968-1997¹].

SEgonds, A.-Ph. (ed.), 2003², Proclus, Sur le Premier Alcibiade de Platon, vol. I-II, Paris [1985-1986¹].

SkLIRIs, D., 2008, “The Theory of Evil in Proclus. Proclus' Theodicy as a Completion of Plotinus' Monism”, Philotheos 8, pp. 137-159.

STEeL, C., 1989, “L'Un et le Bien. Les raisons d'une identification dans la tradition platonicienne”, Revue des Sciences philosophiques et théologiques 73, pp. 69-85.

Steel, C., 1997, “Proclus et Denys : de l'existence du mal”, in: de Andia (1997), pp. 89-116.

STEeL, C., 1998, "Proclus on the Existence of Evil”, Proceedings of the Boston Area Colloquium in Ancient Philosophy 14, pp. 83-102 e 109.

STEeL, C., 2017, “Providence and Evil”, in: d'Hoine, Martijn (2017), pp. 240-257.

Steel, C., et al. (eds.), 2007-2009, Proclus, In Platonis Parmenidem commentaria, vol. I-III, Oxford.

STrobel, B., 2014, Proklos. Tria opuscula. Textkritisch kommentierte Retroversion der Übersetzung Wilhelms von Moerbeke, Berlin-Boston.

Suchla, B. R. (ed.), 1990, Pseudo-Dionysius Areopagita, Corpus Dionysiacum, vol. I: De divinis nominibus, Berlin-New York.

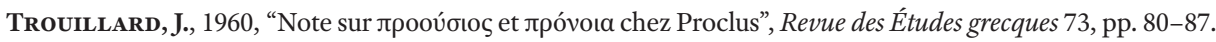

Trouillard, J., 1982, La mystagogie de Proclos, Paris.

Westerink, L. G., 1962, “Notes on the Tria opuscula of Proclus”, Mnemosyne 15, pp. 159-168. 


\section{Ringraziamenti}

Sono grato all'anonimo Reviewer per le pertinenti osservazioni che mi hanno permesso di migliorare questo mio contributo, dei cui limiti resto l'unico responsabile. Ringrazio anche l'Editor-in-Chief di Peitho. Examina Antiqua, Mikołaj Domaradzki, per la cordiale disponibilità che mostra sempre nei miei confronti.

\section{VALERIO NAPOLI}

/ independent researcher, Agrigento, Italy / valerio.napoli@libero.it

\section{Evil Itself and Nothingness in Proclus}

In his reflection on the nature of evil, the Neoplatonic philosopher Proclus affirms that evil itself (to autokakon) is "also beyond the absolute non-being" (epekeina kai tou mèdamōs ontos). With this assumption, he intends to reinforce the thesis of the non-existence of absolute evil, conceived as totally separate from good, and contrasted with the collateral and parasitic existence of evil mixed with good. He thus maintains a distinction between absolute evil and relative evil, conceived with reference to the distinction between absolute non-being (i.e., nothingness) and relative non-being. In Proclus, the thesis of the non-existence of absolute evil is presented as a necessary consequence of the nondualist theory of evil in the sphere of a protology that identifies the first Principle of all things in the primary Good (identical to the supra-essential One), and which aims to reconcile the absolute primacy of the latter with the presence of evil in some orders of reality.

Proclus, Evil, Nothingness, Non-being, Neoplatonism. 